\title{
Concepts, prevalence and characteristics of severe maternal morbidity and near miss in Brazil: a systematic review
}

Josy Maria de Pinho da Silva 1

Sandra Costa Fonseca 2

Marcos Augusto Bastos Dias 3

Aline Silva Izzo 4

Gabrielle Pires Teixeira 5

Pamela Pinto Belfort 6

\footnotetext{
1,2,4-6 Instituto de Saúde Coletiva. Universidade Federal Fluminense. Rua Marques de Paraná, 303 - Anexo - $3^{\circ}$ andar. Niterói, RJ, Brasil. CEP: 24030-215. E-mail: josy.m.pinhos@gmail.com

3 Instituto Nacional de Saúde da Mulher, da criança e do adolescente Fernandes Figueira. Rio de Janeiro, RJ, Brasil.
}

\begin{abstract}
Objective: to analyze frequency, characteristics and causes of severe maternal morbidity (maternal near miss) in Brazil.

Methods: a systematic review on quantitative studies about characteristics, causes, and associated factors on severe maternal morbidity (maternal near miss). The search was done through MEDLINE (maternal near miss or severe maternal morbidity and Brazil) and LILACS (maternal near miss, maternal morbidity). Data were extracted from methodological characteristics of the article, criteria for maternal morbidity and main results. Near miss ratios and indicators were described and estimated.

Results: we identified 48 studies: 37 were on hospital based; six were based on health surveys and five were based on information systems. Different definitions were adopted. Maternal near miss ratio ranged from 2.4/1000 LB to 188.4/1000 LB, depending on the criteria and epidemiological scenario. The mortality rate for maternal near miss varied between $3.3 \%$ and $32.2 \%$. Hypertensive diseases and hemorrhage were the most common morbidities, but indirect causes have been increasing. Flaws in the healthcare were associated to near miss and also sociodemographic factors (non-white skin color, adolescencel age $\geq$ 35 years old, low schooling level).

Conclusions: the frequency of maternal near miss in Brazil is high, with a profile of similar causes to maternal mortality. Inequities and delays in the healthcare were identified as association.
\end{abstract}

Key words Women's health, Complications at pregnancy, Health inequalities 


\section{Introduction}

Women and children health is a worldwide priority, and the losses in puerperal pregnancy period and at childhood are considered devastating for the family and the society. Maternal mortality ratio reflects on the socioeconomic indicators as well as the quality in the offered healthcare, and its decrease in Brazil and in the world was included in the Millennium Goals, and it remains in the Sustainable Development Goals. ${ }^{1}$ The previous goal did not achieve its two-thirds reduction on Maternal Mortality Ratio (MMR), and for Brazil, the challenge is to reduce the MMR from 20/100,000 live births until 2030.1

Despite the high maternal mortality rates, maternal death is an infrequent event in absolute numbers, making local studies and basic causes difficult to understand. In addition, there is a spectrum of morbid conditions between healthy gestation and maternal death ranging from mild to extremely severe conditions. ${ }^{2}$

In this context, the World Health Organization (WHO) defined the criterion of severe maternal morbidity or "maternal near miss" as "a woman who almost died but survived a serious maternal complication during pregnancy, childbirth, or within 42 days of completion of pregnancy." 3 These women have survived severe maternal complications or "life-threatening conditions" due to adequate healthcare services. 3 There is a list of life-threatening conditions (LTC) acknowledged by clinical, laboratorial or even management characteristics that support this classification established by WHO in order to unify the diagnostic criteria. 3

Prior to WHO, there were other criteria for this outcome, ranging from the admission at the Intensive Care Unit to organ dysfunction, with different accuracy measurements. ${ }^{4}$ Life-threatening conditions are the extreme of potential life-threatening conditions (PLTC) or maternal complications and that relates to some organ dysfunction feature. ${ }^{2}$

Several indicators derived from the near miss concept and can be used in research and obstetrical audits. The maternal near miss ratio (MNMR) refers to the number of maternal near miss cases by the number of live births (by 100,000); severe maternal outcomes (or life-threatening condition) includes cases of near miss and maternal death; maternal near miss mortality ratio and maternal death (MNM: $\mathrm{MD}$ ); and the mortality rate (MR), which refers to the proportion of maternal deaths from the total sever outcomes. The latter two reflect the effectiveness on care in preventing a severe case evolving in death, and expecting a high MNM: MD and a low MR. 3

In addition to women's commitment, severe maternal morbidity/maternal near miss has an impact on fetal and neonatal outcomes, including neonatal near miss. 5

We have not identified reviews on severe maternal morbidity/near miss in Brazil, and the most recent international review published in 2013, included a few Brazilian studies. ${ }^{6}$ Considering this gap and the relevance that deaths and other maternal outcomes such as abortion, hypertensive diseases, hemorrhages and infections have an effect on women and children's health, the objective of this article was to review Brazilian medical literature on maternal near miss.

\section{Methods}

A systematic review of the literature on severe maternal morbidity/maternal near miss in Brazil was carried out, without date restriction and completed search was in October 2016.

Regarding eligibility criteria, we considered two main approaches for articles inclusion on: descriptive studies (description of maternal morbidity/nearmiss rates, description of causes); studies on factors associated to maternal morbidity/near miss outcomes (cross-sectional or longitudinal).

Case reports, studies with specific pathological morbidity groups not directly related to maternal morbidity and mortality and studies where maternal morbidity was the exposure variable and not an outcome, were excluded.

Review studies were initially included to widen the identification of original studies and subsequently were excluded. We also excluded letters, editorials, dissertations and theses, prioritizing fulltext articles already published in scientific journals. We adopted as an exclusion criterion articles in which Brazil was not the only country addressed, in order to emphasize national approaches on the theme.

The bibliographic search was performed using LILACS databases (through Virtual Health Library) and MEDLINE (through PubMed), without language restriction. The terms severe maternal morbidity and near miss still do not exist as descriptors in scientific literature bases.

In LILACS, the search strategy was performed in two stages (the use of Boolean operator OR joining the two terms resulted in a fewer number of articles) using the terms: severe maternal morbidity and maternal near miss, at each stage. For 
MEDLINE, the strategy was: (near miss or severe morbidity) and maternal and Brazil.

The search was performed independently by JMPS (first author) and SCF (second author), and the disagreements were solved by consensus. An additional manual search in the bibliographic references of the articles included was carried out.

Initially the titles of the articles were evaluated and the titles rejected by both researchers were excluded. The titles approved by at least one of the authors went through a second stage, reading the abstracts. In this stage, the studies with abstracts approved by both authors were included.

From the selected abstracts, the full-text articles were read to confirm eligibility and to collect relevant information. For the reading and synthesis stages, besides the first and second authors, other authors have participated (academics in their last periods in Medicine, with interest in the area of Obstetrics). Each article was read by at least two authors (always the first or second author, plus a third one) independently, and the disagreements were solved by consensus. The reasons for the final exclusion are listed in the flowchart, as recommended by PRISMA. ${ }^{7}$

The data - author, location, population characteristics, guidelines, data source, severe maternal morbidity/near miss criteria and the main results were collected according to a pre-established spreadsheet. At least two of the authors read and analyzed all the articles. Among the results, the following quantitative indicators were highlighted: ratio or near miss incidence, MNM/MD and mortality rate. When the indicators were not described, but contained the necessary information for its calculation, it was estimated and added to the results in the review.

The methodological quality was not an inclusion/exclusion criterion in the analysis, considering that the purpose was to analyze a broad spectrum of studies on severe maternal morbidity, and to point out aspects referring to the methodology used. An experienced obstetrician on the subject also evaluated the summarization and the analysis of articles.

In order to organize the categories of the articles by prioritizing the data source, as proposed by Cecatti et al. 8 "studies on hospital population, studies on type of surveys and studies based on information systems".

This review is part of a study approved by the Ethics Committee of the Universitário Antônio Pedro on November 14, 2016, document number 1826053 , to study the relation between near miss and neonatal outcomes.

\section{Results}

209 titles were identified in the MEDLINE search and 113 (considering the two combinations) in LILACS. After the exclusion of duplicates, selection and full reading of the articles, 48 studies for the systematic review were selected (Figure 1).

Tables 1 to 4 show that the studies are organized according to the data source type: hospital based (local and national), population surveys and information systems. Each category was preserved the chronological order of the publication, although there are some differences between this date and the moment of the implementation of the studies.

The 48 studies found were divided in: 37 hospital based; six were based on health surveys and five were based on information systems. The total number of the articles, $30(62.5 \%)$ were published in international journals, all in English, 22 belonged in the Gynecology and Obstetrics and Reproductive Health areas. Among the 18 national publications, the most frequent journals were on Public Health (8), followed by Internal Medicine (7) and GynecologyObstetrics (3). Of the national internal medicine articles, four were exclusively published in English.

\section{Local hospital based studies}

In this category (Table 1), 22 studies were identified $9-30$ originated from the Southeast (11) and Northeast (11) regions. Of these, 12 were crosssectional studies, in which nine were only descriptive studies. The case-control type was a design of four studies and five of cohort studies, in which three were retrospective. One study was longitudinal, but just descriptive.

Most of the studies used the terminology "maternal near miss". Regarding to the criteria used for the definitions of near miss and severe maternal morbidity, $10(44 \%)$ used the WHO criteria, 10 $(40 \%)$ of Waterstone, 31 eight (32\%) of Mantel, 32 three $(16 \%)$ for the ICU admission, two $(8 \%)$ the criteria proposed by Reichenheim et al., ${ }^{4}$ two (8\%) used life-threatening conditions as a criteria and only one used Geller's criteria. ${ }^{33}$ It is worth mentioning that most of the studies included more than one criterion in their analysis. Morse et al. ${ }^{15}$ study compared three criteria, becaming the first one to use the WHO criteria in Brazilian studies.

Considering studies that adopted the WHO criteria, management and laboratorial criteria were the most prevalent, each being the most prevalent in two studies. Severe preeclampsia was the most common criterion identifier ever. 
Figure 1

Flowchart on the selection of articles.

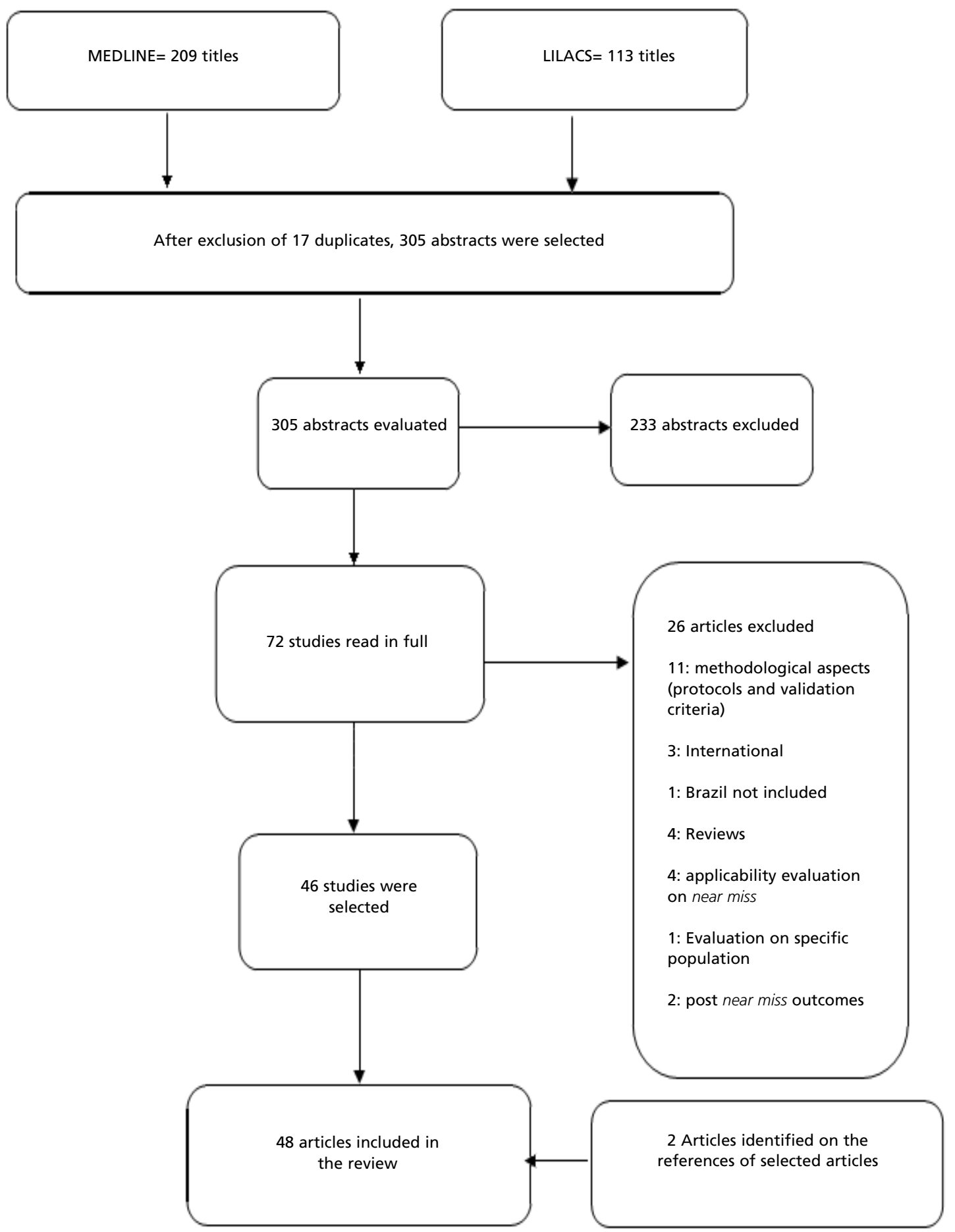




\begin{tabular}{|c|c|c|c|c|c|c|}
\hline $\begin{array}{l}\text { Author and } \\
\text { year of article }\end{array}$ & $\begin{array}{l}\text { Study } \\
\text { location }\end{array}$ & $\begin{array}{l}\text { Studied } \\
\text { Period }\end{array}$ & $\begin{array}{l}\text { Type of study/ } \\
\text { source/ analysis }\end{array}$ & Population & Criteria & Results \\
\hline Souza et al.9 2005 & $\begin{array}{l}\text { Campinas, } \\
\text { São Paulo }\end{array}$ & $\begin{array}{l}\text { From July } \\
2003 \text { until } \\
\text { June } 2004\end{array}$ & $\begin{array}{l}\text {-Case-control } \\
\text {-Bivariate analysis }\end{array}$ & $\begin{array}{l}\text { - } 124 \text { women with } \\
\text { severe maternal } \\
\text { morbidity at a } \\
\text { University maternity }\end{array}$ & $\begin{array}{l}\text {-Mantel et } \\
\text { al.32 or } \\
\text { Waterstone } \\
\text { et al.31 } \\
\text {-Additional: } \\
\text { Geller et al.33 }\end{array}$ & $\begin{array}{l}\text {-Severe maternal morbidity (SMM): } 124 \text { cases (Mantel or Waterstone } \\
\text { criteria). SMM ratio: } 42 / 1,000 \text { childbirths } \\
\text { - Near miss ratio (Geller } 33 \text { criteria): } 6,8 / 1,000 \text { childbirths; } 20 \text { women with } \\
\text { very severe maternal morbidity (Cases) and } 104 \text { controls "with others } \\
\text { severe morbidities" (Control) } \\
\text { - Previous abortion was the only association with SMM (OR=3.41; } \\
95 \% \mathrm{Cl}=1.08-10.79 \text { ). } \\
\text {-Hypertension was the only clinical condition most frequent for SMM, } \\
\text { while hemorrhage predominated on the near miss group }\end{array}$ \\
\hline Souza et al.10 2007 & $\begin{array}{l}\text { Campinas, } \\
\text { São Paulo }\end{array}$ & $\begin{array}{l}\text { From July } \\
2003 \text { until } \\
\text { June } 2004\end{array}$ & $\begin{array}{l}\text {-Cross-sectional } \\
\text { descriptive } \\
\text {-Daily visits: deli- } \\
\text { very rooms, ICU, } \\
\text { Ward. Review on } \\
\text { medical files after } \\
\text { hospital leave. }\end{array}$ & $\begin{array}{l}-2929 \text { childbirths in } \\
\text { a university mater- } \\
\text { nity }\end{array}$ & $\begin{array}{l}\text {-Mantel et } \\
\text { al.32 or } \\
\text { Waterstone } \\
\text { et al.31 }\end{array}$ & $\begin{array}{l}\text {-Total: } 124 \text { cases of SMM (SMM ratio= } 42 / 1000 \text { childbirths). - } 2 \\
\text { maternal deaths. MNM/MD ratio } 62 \\
\text { - Waterstone's criteria }=86 \text { (SMM ratio }=38 / 1000) \text {, which pre- } \\
\text { eclampsia was the most frequent; }- \text { Mantel's criteria }=62 \text { (SMM ratio= } \\
31 / 1000) \text {, which ICU admission was the most frequent } \\
\text { - Most common conditions }=\text { Hypertension }(57.3 \%) \text {, non-obstetrics } \\
(21 \%) \text { and hemorrhage }(13.7 \%)\end{array}$ \\
\hline Amorim et al.11 2008 & $\begin{array}{l}\text { Recife, } \\
\text { Pernambuco }\end{array}$ & $2003-2007$ & $\begin{array}{l}\text {-Cross-sectional } \\
\text { descriptive } \\
\text {-Review on medi- } \\
\text { cal files }\end{array}$ & $\begin{array}{l}-291 \text { women with } \\
\text { near miss criterion } \\
\text { admitted in the } \\
\text { obstetrics ICU }\end{array}$ & $\begin{array}{l}\text {-Mantel et } \\
\text { al.32 }\end{array}$ & $\begin{array}{l}\text { - Of the } 291=\text { hypertension }(78.4 \%) \text { was the main cause for admission, } \\
\text { followed by hemorrhage } \\
\text { - Most common clinical diseases: heart diseases }(5.8 \%) \text {, chronic arterial } \\
\text { hypertension }(5.1 \%) \text {, chronic liver diseases }(3 \%) \text { and diabetes mellitus } \\
(2.4 \%) \\
\text { - Eclampsia was present in } 38.8 \% \text {, HELLP syndrome in } 28,2 \% \text { and } \\
\text { hemorrhagic shock in } 27.1 \% \text { of the patients } \\
\text { - Most common invasive procedures: blood transfusion ( } 36 \%) \text {, } \\
\text { profound venous puncture }(13.4 \%) \text {, vasoactive drugs (10.8\%) and } \\
\text { assisted mechanical ventilation }(9.1 \%)\end{array}$ \\
\hline
\end{tabular}

PLTC= Potential life-threatening conditions; SMO= severe maternal outcome=MNM+MD; HA= hypertension; HELLP= hemolysis elevated liver enzymes low platelet; $C \mathrm{Cl}=\mathrm{confinues}$ interval; $\mathrm{MD}=$ maternal death $\mathrm{SMM}=$ severe maternal morbidity; $\mathrm{MNM}=$ maternal near miss; $\mathrm{LB}=$ live births; WHO= World Health Organization; $\mathrm{OR}=0 \mathrm{dds}$ ratio; $\mathrm{MMR}=$ maternal mortality ratio; $S M M R=$ severe maternal morbidity ratio; $P R=$ prevalence ratio; $R R=$ relative risk; SUS= Public Health System; ICU= intensive care unit. 
Local hospital based studies.

\begin{tabular}{lccccc}
\hline $\begin{array}{l}\text { Author and } \\
\text { year of article }\end{array}$ & $\begin{array}{c}\text { Study } \\
\text { location }\end{array}$ & $\begin{array}{c}\text { Studied } \\
\text { Period }\end{array}$ & $\begin{array}{c}\text { Type of study/ } \\
\text { source/ analysis }\end{array}$ & Population & Criteria \\
\hline
\end{tabular}

Luz et al.12 $2008 \quad$ Campinas,

São Paulo

-Cross-sectional
-Review on medi-

2207 childbirths

-Mantel et al.32 - SMM = 114 cases ( 15 of extremely severe morbidity ESMM and 99 of cal files

SMM

al. 31

-Non-adjusted

prevalence ratio

- ESMM rate $=6.8 / 1,000$ childbirths; Rate of others materna morbidities $=44.9 / 1,000$ childbirths

- Most common conditions of ESMM= post-childbirth hemorrhage $(46.7 \%)$, insufficiency respiratory $(13.4 \%)$, hypertension $(13.3 \%)$ and pre-childbirth hemorrhage $(13.3 \%)$; without any association with the variables in the study

$\begin{array}{ll}\text { Oliveira-Neto et al.,13 } & \text { Campinas, } \\ 2009 & \text { São Paulo }\end{array}$

São Paulo

$2002-2007$

673 women in the ICU obstetric at the

U admission - Of the 673 women, 18 cases of MD and 655 of SMM - SMM ratio $=46.6 / 1,000 \mathrm{LB} ; \mathrm{MMR}=124 / 100,000 \mathrm{LB}$

-Causes of MNM and SMM/MD ratio= Hypertension: 322 cases, ratio 321:1 / Hemorrhage: 92 cases, ratio 30:1 / Infections: 17 cases, ratio 6:1 Non-obstetrics: 230 cases, ratio 25:1

-Interventions/procedures associated to death: reanimation mechanical ventilation, vasoactive drugs use, cardioversion, blood transfusion

F $r \circ m$-Cross-sectional October descriptive

2005 until -Daily collection December of medical files 2005 and hospital registration

-MD committee evaluation

-All cases of severe -Mantel'et al.32 - MNM total cases= $95 /$ Maternal death total $=4$ MNM ratio $=$ acute maternal adapted

morbidity/ near miss criteria

in the city.

$\begin{array}{ll}\text {-Waterstone et } & (17.9 \%) \\ \text { al. } 31 & \text { - Delays }\end{array}$ (he use of magnesium sulfate and post-childbirth hemorrhage prophylaxis)

PLTC= Potential life-threatening conditions; $S M O=$ severe maternal outcome=MNM+MD; HA= hypertension; HELLP= hemolysis elevated liver enzymes low platelet; $C l=$ confidence interval; $\mathrm{MD}=$ maternal death; $\mathrm{SMM}=$ severe maternal morbidity; $\mathrm{MNM}=$ maternal near miss; $\mathrm{LB}=$ live births; WHO= World Health Organization; OR= odds ratio; MMR= maternal mortality ratio; $S M M R=$ severe maternal morbidity ratio; $P R=$ prevalence ratio; $R R=$ relative risk; $S U S=$ Public Health System; ICU= intensive care unit. 
Local hospital based studies.

\begin{tabular}{|c|c|c|c|c|c|c|}
\hline $\begin{array}{l}\text { Author and } \\
\text { year of article }\end{array}$ & $\begin{array}{c}\text { Study } \\
\text { location }\end{array}$ & $\begin{array}{c}\text { Studied } \\
\text { Period }\end{array}$ & $\begin{array}{l}\text { Type of study/ } \\
\text { source/ analysis }\end{array}$ & Population & Criteria & Results \\
\hline Morse et al.,15 2011 & $\begin{array}{l}\text { Niterói, Estado } \\
\text { do Rio de Janeiro }\end{array}$ & 2009 & $\begin{array}{l}\text {-Cross-sectional } \\
\text { descriptive } \\
\text {-Review on medi- } \\
\text { cal files }\end{array}$ & $\begin{array}{l}-1.554 \text { childbirths in } \\
\text { a referral public } \\
\text { maternity hospital } \\
\text { for, high-risk in the } \\
\text { metropolitan region } \\
\text { II of Rio de Janerio }\end{array}$ & $\begin{array}{l}\text {-Mantel et } \\
\text { al.32 } \\
\text {-Waterstone } \\
\text { et al.31 } \\
\text {-WHO3 }\end{array}$ & $\begin{array}{l}\text { - MNM cases, considering any criteria: } 89 \text { cases; by WHO criteria, } 10 \\
\text { cases. Three cases of MD } \\
\text { - MNMR }=- \text { WHO }-9.4 / 1,000 \text { live births; Waterstone }-81.4 / 1,000 \text { live } \\
\text { births; - Mantel }-13.1 / 1,000 \text { live births } \\
\text {-Mortality rate }(\text { WHO }=23 \% \text {; MNM/MD ratio }=3.3 \\
\text { Most frequent markers were: severe preeclampsia }(68.5 \%) \text {, severe } \\
\text { hemorrhage }(19.1 \%) \text { and ICU admission }(10.1 \%)\end{array}$ \\
\hline Moraes et al.,16 2011 & $\begin{array}{l}\text { São Luiz, } \\
\text { Maranhão }\end{array}$ & 2009-2010 & $\begin{array}{l}\text {-Longitudinal des- } \\
\text { criptive } \\
\text {-Search on medi- } \\
\text { cal files and inter- } \\
\text { views with health } \\
\text { professionals at } \\
\text { the health units } \\
\text {-Fisher test, } \chi^{2}, t- \\
\text { Student test and } \\
\text { Mann-Whitney }\end{array}$ & - 8493 childbirths & $\begin{array}{l}\text {-Mantel et } \\
\text { al.32 } \\
\text {-Waterstone } \\
\text { et al. } 31\end{array}$ & $\begin{array}{l}\text { - } 127 \text { women presented one of the analyzed criteria } \\
\text {-Incidence rate of severe maternal morbidity= Total: } 15 / 1,000 \\
\text { childbirths; Waterstone: } 14.1 / 1,000 \text { childbirths Mantel: } 3.4 / 1,000 \\
\text { childbirths } \\
\text { - At admission, } 84 \% \text { of the women presented hypertension disorders, } \\
11.4 \% \text { hemorrhagic disorders, } 2.5 \% \text { infected abortion and } 1.6 \% \text { other } \\
\text { causes } \\
\text {-Significant association with ESMM cases: longer hospitalization time } \\
(p \text {-value }=<0.001) \text { e hemorrhage ( } p \text {-value }=<0.01)\end{array}$ \\
\hline Lotufo et al., 17 2012 & $\begin{array}{l}\text { Limeira, São } \\
\text { Paulo }\end{array}$ & $2004-2007$ & $\begin{array}{l}\text {-Cross-sectional } \\
\text {-Multivariate re- } \\
\text { gression }\end{array}$ & $\begin{array}{l}-158 \text { women admit- } \\
\text { ted at an obstetric } \\
\text { ICU at a general } \\
\text { teaching hospital }\end{array}$ & $\begin{array}{l}-\mathrm{WHO} \\
-\mathrm{ICU} \\
\text { admission }\end{array}$ & $\begin{array}{l}\text { ICU admission immediately after childbirth }(87 \%) \\
\text { - From the total, } 5 \mathrm{MD}, 43 \mathrm{MNM}, 110 \mathrm{PLTC} \\
\text { - MNM ratio }=4.4 / 1,000 \text { live births; Mortality rate }=10.6 \% \text { (higher for } \\
\text { clinical-surgical conditions and infections) } \\
\text { - MNM/MD }=8.6 \\
\text { - Most common management criteria, outlining vasoactive drugs, } \\
\text { hysterectomy and blood transfusion; among the laboratory, the } \\
\text { PaO2/Fio2<200 ratio was the most present and, among the clinical } \\
\text { criteria, shock. } \\
\text { - The only association between MNM and MD: cesarean section } \\
(\mathrm{OR}=0.03 ; \mathrm{Cl}=0.002-0.49 \text { ) }\end{array}$ \\
\hline
\end{tabular}


Local hospital based studies.

\begin{tabular}{|c|c|c|c|c|c|c|}
\hline $\begin{array}{l}\text { Author and } \\
\text { year of article }\end{array}$ & $\begin{array}{l}\text { Study } \\
\text { location }\end{array}$ & $\begin{array}{c}\text { Studied } \\
\text { Period }\end{array}$ & $\begin{array}{l}\text { Type of study/ } \\
\text { source/ analysis }\end{array}$ & Population & Criteria & Results \\
\hline Moraes et al.,18 2013 & $\begin{array}{l}\text { São Luiz, } \\
\text { Maranhão }\end{array}$ & $2009-2010$ & $\begin{array}{l}\text {-Case-control } \\
\text {-Health profes- } \\
\text { sionals' report }\end{array}$ & $\begin{array}{l}\text {-Two high-risk ma- } \\
\text { ternities and two } \\
\text { referral obstetric ICU } \\
-122 \text { MNM cases at } \\
\text { the ICU and } 244 \\
\text { controls (mater- } \\
\text { nities) }\end{array}$ & $\begin{array}{l}\text {-Mantel et } \\
\text { al.32 } \\
\text {-Waterstone } \\
\text { et al.31 }\end{array}$ & $\begin{array}{l}\text { Total of cases: } 122 \\
\text { - } 66.4 \% \text { presented severe pre-eclampsia, } 11.5 \% \text { eclampsia, } 11.4 \% \text { obstetric } \\
\text { hemorrhage, } 5.7 \% \text { HELLP syndrome, } 2.5 \% \text { infected abortion, } 1.6 \% \text { pre- } \\
\text { eclampsia overlapped chronic hypertension and } 1.6 \% \text { obstetrics } \\
\text { complications } \\
\text { - Statistically significant association with SMM: age } \geq 35 \text { years old }(O R=3.11) \text {, } \\
\text { previous hypertension }(O R=2.52),<4 \text { prenatal consultations (OR=1.89) }\end{array}$ \\
\hline
\end{tabular}

Lobato et al.,19 2013 Rio de Janeiro, Estado do Rio de Janeiro

2008

\section{8}

-Cross-sectional descriptive -Review on dical files
Unive hospital
-Waterstone et al. 31 $-\mathrm{WHO}^{3}$ -Reichenheim et al.,4 2009 criteria

Total of cases (considering any criteria): 157

- MNMR were:

33.2/1,000 live births by WHO; 155.2/1,000 live births by Waterstone 188.4/1,000 live births by Reichenheim criteria 2009

$-2.3 \%$ of the cases presented WHO criteria, $10.8 \%$ Waterstone criteria and 13.2\% Reichenheim criteria 2009

- From 27 cases of WHO, 77.8\% were positive in other classification, 14.8\% were exclusive of WHO (thrombocytopenia)

- From 25 cases classified as exclusively by Reichenheim 2009, 80\% presented severe hypertension, $68 \%$ were admitted to ICU, $20 \%$ received blood transfusion, $4 \%$ had pulmonary edema and $4 \%$ developed hemorrhage

- The cases classified by Reichenheim 2009 and by Waterstone (105), 94.3\% were pre-eclampsia, eclampsia and HELLP syndrome

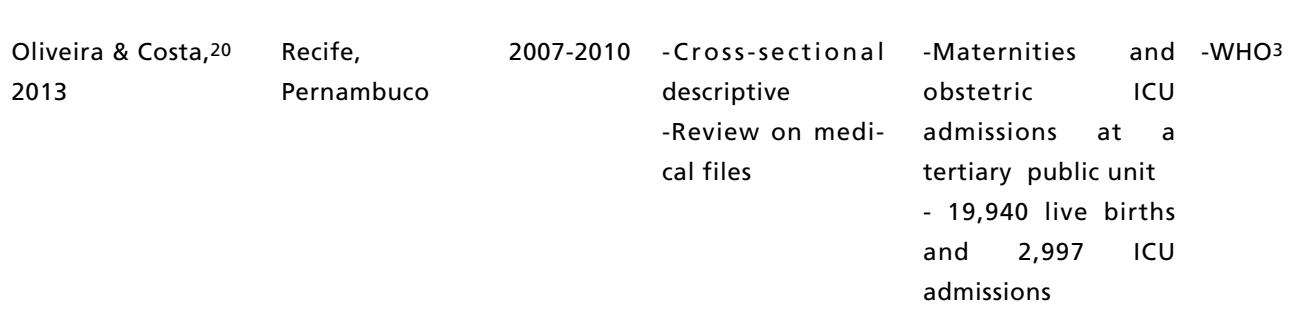

Near miss cases $=255$

- MNMR= $12.8 / 1,000$ live births

- Hypertensions disorders occurred in $62.7 \%$, mainly severe pre-eclampsia $(49 \%)$ and eclampsia $(13.7 \%)$

- The most frequent infection was endometritis $(25.1 \%)$, followed by pneumonia (19.6\%)

$-35.3 \%$ of postpartum hemorrhage was also identified

- Laboratory criteria was present in $59.6 \%$ of the participants, while clinical and management criteria occurred in $50.2 \%$ and $49 \%$, respectively. 
Local hospital based studies.

\begin{tabular}{lcccccc}
\hline $\begin{array}{l}\text { Author and } \\
\text { year of article }\end{array}$ & $\begin{array}{c}\text { Study } \\
\text { location }\end{array}$ & $\begin{array}{c}\text { Studied } \\
\text { Period }\end{array}$ & $\begin{array}{c}\text { Type of study/ } \\
\text { source/ analysis }\end{array}$ & Population & Criteria & Results \\
\hline Amorim et al.,21 & Recife, & $2008-2009$ & -Cohort & -500 severe pre- & -PLTC & - Global PLTC according to the type of childbirth: cesarean section -
\end{tabular}

Amorim et al.,21 Recife,

2014

Pernambuco

2008-2009

\section{-Cohort}

eclampsia patients,

RR complication without other clini-

according to

type of childbirth

mitted at a tertiary

-Multivariate ana- hospitals

lysis: OR of com-

plication accor-

ding to the type

of childbirth

Galvão et al.,22 2014 Sergipe

2011-2012 -Case-control in-

cidence

-Medical files, pre-

-Two referral maternities for the whole

natal care cards

$-16,243$ live births

and interviews
-PLTC - Global PLTC according to the type of childbirth: cesarean section $54 \%$ and vaginal $-32.7 \%$

Conditions associated to cesarean section in women with severe preeclampsia: RR $(95 \% \mathrm{Cl})$

- Post-childbirth hemorrhage: RR=9.8 (2.4-39.9)

-Hypertensive crisis: 1.58 (1.22-2.06)

Associated factors with PLTC in 500 women:

Cesarean section - OR=1,91 (1.52-4.57)

Pre-childbirth HELLP syndrome- OR=3.91 (1.55-9.88)

$-\mathrm{WHO}$

-Total of SMM (or PLTC): 1,102 cases

-Near miss cases: 77/Maternal deaths: 17

- MNMR: 4.7/1,000 live births; SMMR: 67.8/1,000 live births

Mortality rate: $18 \%$; MNM/MD ratio $=4.5$

- Most frequent morbidities: $67.5 \%$ hypertensive disorders, $15.4 \%$ hemorrhagic disorders and $61.7 \%$ one of the critical interventions defined by WHO;

- Most frequent criteria: $87.1 \% \geq 1$ management criteria, $41.4 \% \geq 1$ clinical criteria and $21.4 \% \geq 1$ laboratorial criteria

Statistically significant association to near-mis: previous abortion $(\mathrm{OR}=2.68)$, previous cesarean section $(\mathrm{OR}=1.64)$ and current $(2.36)$ and conscience alteration $(\mathrm{OR}=15.18)$

PLTC= Potential life-threatening conditions; $S M O=$ severe maternal outcome=MNM+MD; HA= hypertension; HELLP= hemolysis elevated liver enzymes low platelet; $C l=c$ continues

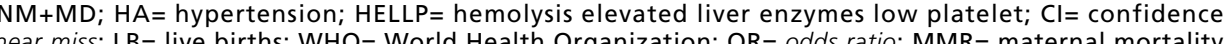
ratio; SMMR= severe maternal morbidity ratio; $P R=$ prevalence ratio; $R R=$ relative risk; SUS= Public Health System; ICU= intensive care unit. 
Local hospital based studies.

\begin{tabular}{lccl}
\hline $\begin{array}{l}\text { Author and } \\
\text { year of article }\end{array}$ & $\begin{array}{c}\text { Study } \\
\text { location }\end{array}$ & $\begin{array}{c}\text { Studied } \\
\text { Period }\end{array}$ & $\begin{array}{c}\text { Type of study/ } \\
\text { source/ analysis }\end{array}$ \\
\hline Menezes et al.,23 & Sergipe & $2011-2012$ & $\begin{array}{l}\text {-Cross-sectional } \\
\text { descriptive } \\
2015\end{array}$ \\
& & -Hospital files
\end{tabular}

Population Criteria Results

$\begin{array}{llllllll}-20,435 \text { women } & - \text { Waterstone et al.31 } & \text { MNMR }= & \text { Reichenheim et } & \text { al.4 } & 2009= & 59 / 1,000 & \text { LB } \\ \text { admitted in two } & - \text { WHO3 }^{3} & \text { Waterstone et al.31 }=24.8 / 1,000 \text { LB } & & & & & \end{array}$
admitted in two -WHO3 Waterstone et al.31= 24.8/1,000 LB $\mathrm{m}$ aternities, -Reichenheim et al. 4 WHO3 $=4.7 / 1,000$ LB

1,196 with po- 2009 criteria $\quad-6.4 \%$ of the cases were compatible with WHO criteria, $33,8 \%$ tential life compatible with Waterstone and $80,2 \%$ compatible with Reichenheim threatening 2009

conditions - Of the 77 WHO cases, 4 were exclusive, 72 were positive also by (PLTC) Reichenheim 2009 and 37 also positive by Waterstone:

- The most frequent criteria according to Reichenheim 2009 were: $54.8 \%$ severe hypertension, $28.3 \%$ blood transfusion and $7.5 \% \mathrm{ICU}$ admission

- The most frequent criteria according to Waterstone were: $71.6 \%$ severe pre-eclampsia, $13 \%$ eclampsia and $7.6 \%$ HELLP syndrome

$\begin{array}{ll}\text { Pacheco et al.,24 } & \text { Vale de São } \\ 2014 & \text { Francisco, } \\ & \text { Pernambuco }\end{array}$

2011 -Retrospective cohort - 2,291 women -Prenatal care cards, medical files -Multivariate analysis

-Logistic regression

$\begin{array}{llll}\text { Souza et al.,25 } 2015 \quad \text { Natal, Rio } & 2013-2014 & \text {-Cross-sectional } \\ & \text { Grande do Norte } & \text {-Review on medical } \\ & & \text { files } \\ & -\chi^{2} \text { tests }\end{array}$
-Waterstoneet al.31 - There were more near miss cases when woman presented= 1) -Geller et al.33 hypertensive disorders $(50.0 \%) ; 2$ ) severe sepsis $(23.8 \%) ; 3)$ severe hemorrhage $(21.4 \%)$
- Clinical conditions with significant relative risk for MNM: first childbirth $(O R=3.1)$, hypertensive gestational disorders $(O R=8.0)$ and cesarean section $(\mathrm{OR}=39.2)$

PLTC= Potential life-threatening conditions; $\mathrm{SMO}=$ severe maternal outcome=MNM+MD; $\mathrm{HA}=$ hypertension; $\mathrm{HELLP}=$ hemolysis elevated liver enzymes low platelet; $\mathrm{Cl}=\mathrm{confidence}$ interval; $\mathrm{MD}=$ maternal death; $\mathrm{SMM}=$ severe maternal morbidity; $\mathrm{MNM}=$ maternal near miss; $\mathrm{LB}=$ live births; $\mathrm{WHO}=\mathrm{W}$.rld Health Organization; $\mathrm{OR}=$ odds ratio; $\mathrm{MMR}=$ maternal mortality interval; $M D=$ maternal death; $S M M=$ severe maternal morbidity; $M N M=$ maternal near miss; $L B=$ live births; $W H O=$ World Health Organization
ratio; $S M M R=$ severe maternal morbidity ratio; $P R=$ prevalence ratio; $R R=$ relative risk; $S U S=$ Public Health System; ICU= intensive care unit. 
Local hospital based studies.

\begin{tabular}{|c|c|c|c|c|c|c|}
\hline $\begin{array}{l}\text { Author and } \\
\text { year of article }\end{array}$ & $\begin{array}{l}\text { Study } \\
\text { location }\end{array}$ & $\begin{array}{l}\text { Studied } \\
\text { Period }\end{array}$ & $\begin{array}{l}\text { Type of study/ } \\
\text { source/ analysis }\end{array}$ & Population & Criteria & Results \\
\hline $\begin{array}{l}\text { Oliveira \& Costa,26 } \\
2015\end{array}$ & $\begin{array}{l}\text { Recife, } \\
\text { Pernambuco }\end{array}$ & 2007-2010 & $\begin{array}{l}\text {-Cross-sectional } \\
\text { descriptive } \\
\text {-Review on medi- } \\
\text { cal files }\end{array}$ & $-2,997$ & -WHO3 & $\begin{array}{l}\text { - MNMR: } 12.8 / 1,000 \text { LB } \\
\text { The main disorders presented were: hypertensive, hemorrhagic and } \\
\text { infectious } \\
\text {-Among the hypertensive disorders cases: } 42.3 \% \text { severe pre-eclampsia, } \\
13.7 \% \text { eclampsia and } 6.7 \% \text { gestational aggravated chronic hyperten- } \\
\text { sion; HELLP syndrome in } 41.2 \% \text { of the participants } \\
-59.6 \% \text { presented one or more laboratorial criteria, } 50.2 \% \text { presented } \\
\text { one or more clinical criteria and } 49 \% \text { presented one or more of } \\
\text { management criteria } \\
\text { - Clinical and laboratorial criteria appeared mostly during pregnancy } \\
\text { (42.2 clinical and } 57.9 \% \text { laboratorial), while the management criteria } \\
\text { occurred mainly in postpartum }(45.6 \%)\end{array}$ \\
\hline Madeiro et al. 272015 & Teresina, Piauí & 2012-2013 & $\begin{array}{l}\text {-Cross-sectional } \\
\text { descriptive } \\
\text {-Review on medi- } \\
\text { cal files } \\
\text {-Multivariate ana- } \\
\text { lysis }\end{array}$ & $-5,841$ & $-\mathrm{WHO}^{3}$ & $\begin{array}{l}\text { - MNMR: } 9.6 / 1,000 \text { LB; - SMMR: } 11.3 / 1,000 \text { LB } \\
\text { - MNM/MD ratio: 5.6:1; - Mortality rate: } 15.2 \% \\
\text { - Main determinants of severe maternal morbidity (near miss) and } \\
\text { maternal death: hypertension }(86.1 \%) \text {, hemorrhage }(10 \%) \text { and } \\
\text { infectious diseases }(2.9 \%) \text {. Severe pre-eclampsia, eclampsia and HELLP } \\
\text { syndrome were the main causes of maternal near miss } \\
\text {-Association with MNM: caesarean section (OR=6.2) and " } 5 \text { days } \\
\text { hospitalization (OR=6.7) }\end{array}$ \\
\hline $\begin{array}{l}\text { Barbosa et al.,28 } \\
2015\end{array}$ & $\begin{array}{l}\text { Sorocaba, São } \\
\text { Paulo }\end{array}$ & $\begin{array}{c}4 \text { anos } \\
\text { (não } \\
\text { informado) }\end{array}$ & $\begin{array}{l}\text {-Retrospective } \\
\text { cohort }\end{array}$ & $\begin{array}{l}\text { - } 1,501 \text { women in a } \\
\text { referral hospital: } \\
\text { Chronic arterial } \\
\text { hypertension (564), } \\
\text { pre-eclampsia (579), } \\
\text { eclampsia (74) and } \\
\text { pre-eclampsia } \\
\text { eclampsia overlap- } \\
\text { ping chronic arte-rial } \\
\text { hypertension (284) }\end{array}$ & $-\mathrm{WHO} 3$ & 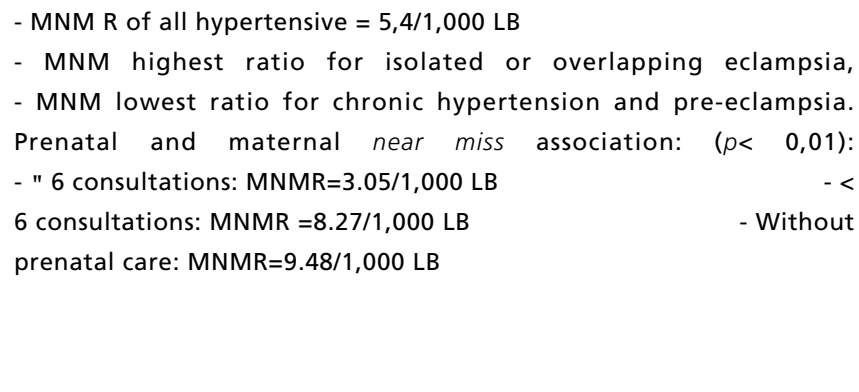 \\
\hline
\end{tabular}


Local hospital based studies.

\begin{tabular}{|c|c|c|c|c|c|c|}
\hline $\begin{array}{l}\text { Author and } \\
\text { year of article }\end{array}$ & $\begin{array}{l}\text { Study } \\
\text { location }\end{array}$ & $\begin{array}{l}\text { Studied } \\
\text { Period }\end{array}$ & $\begin{array}{l}\text { Type of study/ } \\
\text { source/ analysis }\end{array}$ & Population & Criteria & Results \\
\hline Ferreira et al.,29 2015 & $\begin{array}{l}\text { Campinas, São } \\
\text { Paulo }\end{array}$ & $2009-2013$ & $\begin{array}{l}\text {-Cross-sectional } \\
\text { descriptive } \\
\text {-Review on medi- } \\
\text { cal files }\end{array}$ & $\begin{array}{l}-12,771 \text { women ad- } \\
\text { mitted at a referral } \\
\text { University hospital }\end{array}$ & $\begin{array}{c}\text {-ICU } \\
\text { admission }\end{array}$ & $\begin{array}{l}\text {-Near miss incidence: } 50.5 / 1,000 \text { childbirths } \\
\text {-Vaginal section: } 16 / 1,000 \text { childbirths } \\
\text {-Cesarean section: } 91 / 1,000 \text { childbirths } \\
\text {-OR cesarean and near miss association: } 6.24(95 \% \mathrm{Cl} 5.06-7.69)\end{array}$ \\
\hline Vidal et al.,30 2016 & $\begin{array}{l}\text { Barbacena, } \\
\text { Minas Gerais }\end{array}$ & $\begin{array}{c}2014 \text { (Feb- } \\
\text { Aug) }\end{array}$ & $\begin{array}{l}\text {-Case-control } \\
\text {-Interview and } \\
\text { medical files }\end{array}$ & $\begin{array}{l}\text { - } 92 \text { cases and } 184 \\
\text { controls } \\
\text {-Santa Casa Mater- } \\
\text { nity (SUS) }\end{array}$ & $\begin{array}{l}\text {-Life } \\
\text { threatening } \\
\text { conditions }\end{array}$ & $\begin{array}{l}\text {-MNM indicators not described. } \\
\text {-Associated factors: previous hypertension }-\mathrm{OR}=14.3(\mathrm{Cl}=4.5-45.5) \\
\text { cesarean section- } \mathrm{OR}=3.2(1.6-6.3)\end{array}$ \\
\hline
\end{tabular}

PLTC= Potential life-threatening conditions; $S M O=$ severe maternal outcome $=M N M+M D ; H A=$ hypertension; $H E L L P=$ hemolysis elevated liver enzymes low platelet; $C l=$ confidence interval; $\mathrm{MD}=$ maternal death; $\mathrm{SMM}=$ severe maternal morbidity; $\mathrm{MNM}=$ maternal near miss; $\mathrm{LB}=$ live births; WHO= World Health Organization; OR= odds ratio; $\mathrm{MMR}=$ maternal mortality ratio; $S M M R=$ severe maternal morbidity ratio; $P R=$ prevalence ratio; RR= relative risk; SUS= Public Health System; ICU= intensive care unit. 
National hospital based studies / "Born in Brazil" study and "Multicenter study of the National Surveillance Network on Severe Maternal Morbidity".

\begin{tabular}{|c|c|c|c|c|c|c|}
\hline $\begin{array}{l}\text { Author and year } \\
\text { of the article }\end{array}$ & $\begin{array}{l}\text { Study } \\
\text { location }\end{array}$ & $\begin{array}{c}\text { Studied } \\
\text { period }\end{array}$ & $\begin{array}{l}\text { Type of study/ } \\
\text { source/ analysis }\end{array}$ & Population & Criteria & Results \\
\hline Dias et al.5 2014 & -Brazil & 2011-2012 & $\begin{array}{l}\text {-"Born in Brazil" } \\
\text {-Cross-sectional } \\
\text {-Descriptive }\end{array}$ & $\begin{array}{l}\text { - } 23.940 \text { puerperal } \\
\text { had live birth or } \\
\text { stillborn }\end{array}$ & $-\mathrm{WHO}^{3}$ & $\begin{array}{l}\text { - MNM total of cases: } 243 \text { cases }(23,747 \text { cases estimation for the } \\
\text { country) } \\
\text { - MNM ratio: } 10.2 / 1,000 \text { live births } \\
\text { - Among } 243 \text { women, most of them presented only one }(67 \%) \text { or two } \\
\text { (20\%) diagnostic criteria according to WHO } \\
\text { - Most frequent criteria: clinical - respiratory frequency }>40 \text { or }<6 \\
\text { per minute cycles; laboratorial - acute thrombocytopenia (platelets }< \\
50,000) \text {; management - } 5 \text { or more red blood cell units transfusion }\end{array}$ \\
\hline
\end{tabular}

$\begin{array}{llll}\text { Domingues et al.,34 } & \text {-Brazil } \quad 2011-2012 & - \text { "Born in Brazil” } & -23.940 \text { puerperal } \\ 2016 & & \text {-Cross-sectional } & \text { had live birth or } \\ & \text {-Multivariate lo-gistic stillborn } \\ & \text { regression }\end{array}$

$\begin{array}{llll}\text { Santana et al.,37 } & \text {-Brazil } & \text { 2009-2010 } & - \text { "Multicenter stu-dy }- \text { Of the } 9,555 \\ & \text {-27 referral } & \text { of the National women, 237 cases of } \\ & \text { maternities in } & \text { Surveillance Network abortion } \\ & \text { five regions } & \text { on Severe Maternal } \\ & \text { Morbi-dity" } \\ & - \text { Multicenter cross- } \\ & \text { sectional } \\ & - \text { Multivariate ana- } \\ & \text { lyses by Poisson } \\ & \text { regression }\end{array}$

-WHO3 - MNMR for women withoutt prenatal care: 27.9/1,000 LB

- Women 35 years or more presented more clinical or obstetrics complications and had more elective cesarean sections and women with a previous cesarean section presented more complications during pregnancy

-Association with maternal near miss $=$ Prenatal care absence (OR:4.65;1.51-14.31)

-Pilgrimage for 2 or more services (OR:4.49;2.12-9.52)

-Obstetric complications (OR: $9.29 ; 6.69-12.90)$

-Type of childbirth: Elective cesarean section (OR:2.54; $1.67-3.88)$ and forceps (OR:9.37; 4.01-21.91)

-WHO3 -Compared to the 9,318 other women, maternal near miss risk was higher in women who suffered abortion ( $P R=1.93 ; 1.12-3.31)$

-The WHO management criteria were more common in insecure abortion cases.

- Factors associated to abortion complications: previous maternal conditions (sickle-cell anemia, low weight and neoplasia) and previous uterine scar

PLTC (Potential life threatening conditions); SMO (Severe Maternal Outcome=MNM+MD); H1N1 (virus); HA (Hypertension); MD (maternal death); CI (confidence interval); SMM (severe maternal morbidity); MNM (Maternal Near Miss); LB (Live births); WHO (World Health Organization); OR (odds ratio); MMR (maternal mortality ratio); PR (Prevalence ratio); MNMR
(Maternal Near Miss Ratio); HDI (Human Development Index). 
National hospital based studies / "Born in Brazil" study and "Multicenter study of the National Surveillance Network on Severe Maternal Morbidity".

\begin{tabular}{|c|c|c|c|c|c|}
\hline $\begin{array}{l}\text { Author and year } \\
\text { of the article }\end{array}$ & $\begin{array}{l}\text { Study } \\
\text { location }\end{array}$ & $\begin{array}{l}\text { Studied } \\
\text { period }\end{array}$ & $\begin{array}{l}\text { Type of study/ } \\
\text { source/ analysis }\end{array}$ & Population & Criteria \\
\hline $\begin{array}{l}\text { Giordano et al., } 39 \\
2014\end{array}$ & $\begin{array}{l}- \text { Brazil } \\
-27 \text { referral } \\
\text { maternities in } \\
\text { five regions }\end{array}$ & $2009-2010$ & $\begin{array}{l}\text { "Multicenter stu- } \\
\text { dy of the National } \\
\text { Surveillance Net- } \\
\text { work on Severe } \\
\text { Maternal Morbidi- } \\
\text { ty" } \\
\text {-Multivariate ana- } \\
\text { lyses by Poisson } \\
\text { re-gression for } \\
321 \text { cases with } \\
\text { complete infor- } \\
\text { mation. }\end{array}$ & $\begin{array}{l}\text {-Of the } 9,555 \\
\text { women, } 6,280 \text { had } \\
\text { hypertensive disea- } \\
\text { ses and } 426 \text { cases of } \\
\text { eclampsia }\end{array}$ & $-\mathrm{WHO}^{3}$ \\
\hline $\begin{array}{l}\text { Oliveira Jr et al.36 } \\
2014\end{array}$ & $\begin{array}{l}- \text { Brazil } \\
-27 \text { referral } \\
\text { maternities in } \\
\text { five regions }\end{array}$ & $2009-2010$ & $\begin{array}{l}\text {-"Multicenter stu- } \\
\text { dy of the National } \\
\text { Surveillance Net- } \\
\text { work on Severe } \\
\text { Maternal Morbidi- } \\
\text { ty" } \\
\text {-Multivariate ana- } \\
\text { lyses by Poisson } \\
\text { regression to eva- } \\
\text { luate factors rela- } \\
\text { ted to severity } \\
\text { (SMO vs PLTC) in } \\
\text { two age groups }\end{array}$ & $\begin{array}{l}\text { - 9,555 had compli- } \\
\text { cation }\end{array}$ & $-\mathrm{WHO}^{3}$ \\
\hline
\end{tabular}


National hospital based studies / "Born in Brazil" study and "Multicenter study of the National Surveillance Network on Severe Maternal Morbidity".

\begin{tabular}{|c|c|c|c|c|c|c|}
\hline $\begin{array}{l}\text { Author and year } \\
\text { of the article }\end{array}$ & $\begin{array}{l}\text { Study } \\
\text { location }\end{array}$ & $\begin{array}{c}\text { Studied } \\
\text { period }\end{array}$ & $\begin{array}{l}\text { Type of study/ } \\
\text { source/ analysis }\end{array}$ & Population & Criteria & Results \\
\hline $\begin{array}{l}\text { Pacagnela et al., } 46 \\
2014\end{array}$ & $\begin{array}{l}- \text { Brazil } \\
-27 \text { referral } \\
\text { maternities in } \\
\text { five regions }\end{array}$ & $2009-2010$ & $\begin{array}{l}\text { "Multicenter stu- } \\
\text { dy of the National } \\
\text { Surveillance Net- } \\
\text { work on Severe } \\
\text { Maternal Morbi- } \\
\text { dity" } \\
\text {-Multivariate ana- } \\
\text { lyses by Poisson } \\
\text { regression to eva- } \\
\text { luate factors rela- } \\
\text { ted to the delay }\end{array}$ & $\begin{array}{l}-9,555 \text { had compli- } \\
\text { cation }\end{array}$ & $-\mathrm{WHO}^{3}$ & $\begin{array}{l}\text {-Delays on obstetric care: } 58 \% \text { of some kind;- Related to Users: } 10.2 \% \\
\text {-Access related: } 34.6 \% \text { (Most frequent in the } 9,555 \text { women - absence } \\
\text { or inappropriate prenatal care); - Management quality related: } 25.7 \% \\
\text {-Frequency according to severity: In the PLTC cases : } 52 \% \text {, in the MNN } \\
\text { cases: } 68 \% \text {; in maternal deaths: } 84 \% \\
\text { - Increasing association between delay and severity, especially ir } \\
\text { management - } 23.6 \% \text { for PLTC, } 42.3 \% \text { for MNM and } 65 \% \text { for MD } \\
\text { - Associated factors with delay: Adolescence ( } P R=1.09 ; 1.02-1.15) \text {; non } \\
\text { white skin color ( } P R=1.22 ; 1.07-1.39 \text { ); low schooling ( } P R=1.45 ; 1.15 \\
1.84) \text {, public hospital ( } P R=1.98 ; 1.40-2.79) ; \text { absence of prenatal care } \\
(P R=1.66 ; 1.26-2.19 \text { ) and public prenatal care }(P R=1.31 ; 1.03-1.67)\end{array}$ \\
\hline
\end{tabular}

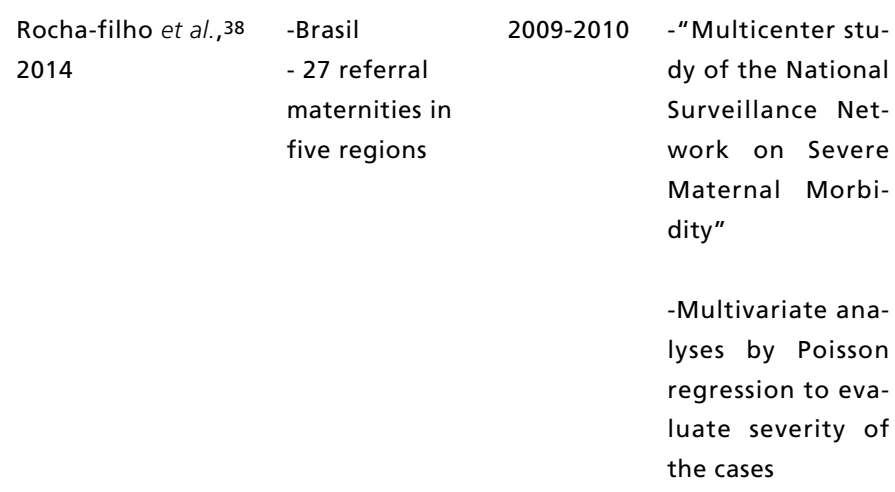

$\begin{array}{lll}\text { Of the } 9,555 & -\mathrm{WHO}^{3} & -\mathrm{PLTC}=286 ; \mathrm{MNM}=25 . \mathrm{MD}=1 \\ \text { women, } 312 \text { had } & & -\mathrm{MNM} \text { ratio }=0.3 / 1,000 \mathrm{LB} ; \mathrm{MNM} / \mathrm{MD} \text { ratio= 25:1; Mortality rate= } \\ \text { ectopic pregnancy } & 3.8 \% \\ \text { cases (EP) } & & - \text { There were no factors associated to severity }\end{array}$

$3.8 \%$

- There were no factors associated to severity

PLTC (Potential life threatening conditions); SMO (Severe Maternal Outcome=MNM+MD); H1N1 (virus); HA (Hypertension); MD (maternal death); Cl (confidence interval); SMM (severe maternal morbidity); MNM (Maternal Near Miss); LB (Live births); WHO (World Health Organization); OR (odds ratio); MMR (maternal mortality ratio); PR (Prevalence ratio); MNMR (Maternal Near Miss Ratio); HDI (Human Development Index). 
National hospital based studies / "Born in Brazil" study and "Multicenter study of the National Surveillance Network on Severe Maternal Morbidity".

\begin{tabular}{|c|c|c|c|c|c|c|}
\hline $\begin{array}{l}\text { Author and year } \\
\text { of the article }\end{array}$ & $\begin{array}{l}\text { Study } \\
\text { location }\end{array}$ & $\begin{array}{l}\text { Studied } \\
\text { period }\end{array}$ & $\begin{array}{l}\text { Type of study/ } \\
\text { source/ analysis }\end{array}$ & Population & Criteria & Results \\
\hline Zanette et al.,40 2014 & $\begin{array}{l}\text {-Brazil } \\
-27 \text { referral } \\
\text { maternities in } \\
\text { five regions }\end{array}$ & $2009-2010$ & $\begin{array}{l}- \text {-"Multicenter stu- } \\
\text { dy of the National } \\
\text { Surveillance Net- } \\
\text { work on Severe } \\
\text { Maternal Morbi- } \\
\text { dity" } \\
\text {-Multivariate ana- } \\
\text { lyses by Poisson } \\
\text { regression }\end{array}$ & $\begin{array}{l}\text { - Of the } 9,555 \text { wo- } \\
\text { men, } 6,315 \text { had } \\
\text { hypertensive disease }\end{array}$ & $-\mathrm{WHO}^{3}$ & 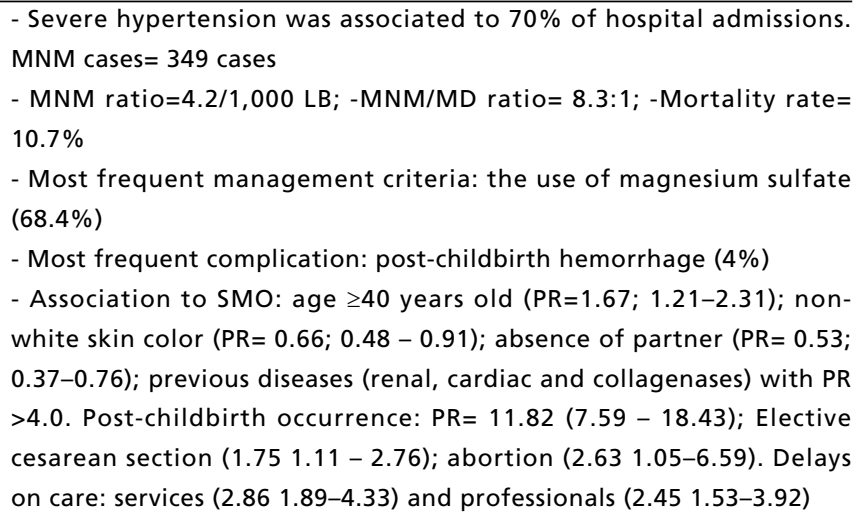 \\
\hline $\begin{array}{l}\text { Campanharo et al., } 45 \\
2015\end{array}$ & $\begin{array}{l}- \text { Brazil } \\
-27 \text { referral } \\
\text { maternities in } \\
\text { five regions }\end{array}$ & $2009-2010$ & $\begin{array}{l}\text {-"Multicenter stu- } \\
\text { dy of the National } \\
\text { Surveillance Net- } \\
\text { work on Severe } \\
\text { Maternal Morbi- } \\
\text { dity" } \\
\text {-Prevalence ratio } \\
\text { adjusted by clus- } \\
\text { ter effect }\end{array}$ & $\begin{array}{l}\text { - Of the } 9,555 \\
\text { women, } 293 \text { cases of } \\
\text { cardiac diseases }\end{array}$ & $-\mathrm{WHO}^{3}$ & $\begin{array}{l}\text {-Heart disease patients' profile: older, low weight, prenatal care at a } \\
\text { public hospital, }<37 \text { weeks for admission. prolonged hospitalization } \\
\text { time. PLTC }=235 \text { cases; MNM }=44 \text { cases } M D=14 \text { cases } \\
\text { MNMR=0.5/1,000 LB; MNMR: MD }=3.1: 1 ; \text { Mortality rate }=24 \% \\
\text {-Predominant clinical }(76 \%) \text { and management }(64 \%) \text { criteria } \\
\text {-Comparing women with heart disease and those without, as for } \\
\text { SMO: PR=2.2 (1.7-2.8); MNM: PR }=2.0(1.3-3.2) ; M D: P R=3.7(2.0-6.9) ;\end{array}$ \\
\hline Ferreira et al..,47 2015 & $\begin{array}{l}\text {-Brazil } \\
-27 \text { referral } \\
\text { maternities in } \\
\text { five regions }\end{array}$ & 2009-2010 & $\begin{array}{l}\text { "Multicenter stu- } \\
\text { dy of the National } \\
\text { Surveillance Net- } \\
\text { work on Severe } \\
\text { Maternal Morbi- } \\
\text { dity" } \\
\text {-Descriptive }\end{array}$ & $\begin{array}{l}\text {-Of the } 9,555 \\
\text { women, those who } \\
\text { had an abortion, } \\
\text { ectopic pregnancy or } \\
\text { missing data were } \\
\text { excluded } \\
\text {-Analysis of } 7,247 \\
\text { puerperals }\end{array}$ & $-\mathrm{WHO}^{3}$ & $\begin{array}{l}\text { - PLTC and SMO according to Robson's classification (RTGCS): } \\
\text { Global: SMO/PLTC=1:12. Groups with the worse performance-7.8.9.10 } \\
\text { (presentation of anomalous, multiple pregnancy and prematurity) } \\
\text { - Among the women who had cesarean: SMO/PLTC }=1: 11 \text {. Groups with } \\
\text { the worse performance }-7.8 .9 .10 \text { and } 3 \text { (multiparas with cephalic } \\
\text { single fetus }>36 \text { weeks) } \\
\text { - Main causes: hypertension }(77.4 \%) \text { and hemorrhage }(21.4 \%) \text {. In } \\
\text { groups } 3,7 \text { and } 9 \text { hemorrhage was }>30 \%\end{array}$ \\
\hline
\end{tabular}

PLTC (Potential life threatening conditions); SMO (Severe Maternal Outcome=MNM+MD); H1N1 (virus); HA (Hypertension); MD (maternal death); Cl (confidence interval); SMM (severe maternal morbidity); MNM (Maternal Near Miss); LB (Live births); WHO (World Health Organization); OR (odds ratio); MMR (maternal mortality ratio); PR (Prevalence ratio); MNMR (Maternal Near Miss Ratio); HDI (Human Development Index). 
National hospital based studies / "Born in Brazil" study and "Multicenter study of the National Surveillance Network on Severe Maternal Morbidity".

\begin{tabular}{|c|c|c|c|c|c|c|}
\hline $\begin{array}{l}\text { Author and year } \\
\text { of the article }\end{array}$ & $\begin{array}{l}\text { Study } \\
\text { location }\end{array}$ & $\begin{array}{c}\text { Studied } \\
\text { period }\end{array}$ & $\begin{array}{l}\text { Type of study/ } \\
\text { source/ analysis }\end{array}$ & Population & Criteria & Results \\
\hline $\begin{array}{l}\text { Rocha Filho et al.41 } \\
2015\end{array}$ & $\begin{array}{l}\text {-Brazil } \\
-27 \text { referral } \\
\text { maternities in } \\
\text { five regions }\end{array}$ & $2009-2010$ & $\begin{array}{l}- \text { "Multicenter stu- } \\
\text { dy of the National } \\
\text { Surveillance Net- } \\
\text { work on Severe } \\
\text { Maternal Morbi- } \\
\text { dity" } \\
\text {-Multivariate ana- } \\
\text { lyses by Poisson } \\
\text { regression }\end{array}$ & $\begin{array}{l}\text {-Of the } 9,555 \\
\text { women, analyses of } \\
767 \text { puerperals had } \\
\text { hemorrhage before } \\
\text { and during child- } \\
\text { birth }\end{array}$ & $-\mathrm{WHO}^{3}$ & $\begin{array}{l}\text {-PLTC: } 613 \text { cases; MNM: } 140 \text { cases; MD: } 14 \text { cases } \\
\text { Mortality rate: } 9 \% \\
\text {-Most common causes: premature placental abruption ( } 50 \% \text { of MNM } \\
\text { and MD cases) } \\
\text {-Among all women with SMO: } 73(52.1 \%) \text { presented one of the clinical } \\
\text { criteria. } 45(32.1 \%) \text { presented one of the laboratorial criteria and } 109 \\
(77.9 \%) \text { presented one of the management criteria } \\
\text { - Associated factors with SMO: age (PR }=1.03 ; 1.01-1.04) \text { and previous } \\
\text { cesarean section (PR }=1.85 ; 1.28-2.66)\end{array}$ \\
\hline $\begin{array}{l}\text { Rocha Filho et al.42 } \\
2015\end{array}$ & $\begin{array}{l}\text {-Brazil } \\
-27 \text { referral } \\
\text { maternities in } \\
\text { five regions }\end{array}$ & $2009-2010$ & $\begin{array}{l}\text {-"Multicenter stu- } \\
\text { dy of the National } \\
\text { Surveillance Net- } \\
\text { work on Severe } \\
\text { Maternal Morbi- } \\
\text { dity" } \\
\text {-Multivariate ana- } \\
\text { lysis by Poisson } \\
\text { regression }\end{array}$ & $\begin{array}{l}\text { - Of the } 9,555 \\
\text { women, } 1,192 \text { puer- } \\
\text { perals had post-chil- } \\
\text { dbirth hemorrhage }\end{array}$ & $-\mathrm{WHO}^{3}$ & $\begin{array}{l}\text { PLTC: } 981 \text { cases; } \mathrm{MNM}=181 \text { cases; } \mathrm{MD}=30 \text { cases; Mortality rate }=15 \% \\
\text { - Of all } 181 \mathrm{MNM} \text { cases, } 78.5 \% \text { presented one of the management } \\
\text { criteria. } 54.7 \% \text { presented one of the clinical criteria and } 35.4 \% \\
\text { presented one of the laboratorial criteria } \\
\text { - Associated factors with } \mathrm{SMO}=\text { maternal age }(\mathrm{PR}=1.03 ; 1.01-1.04) \text {; } \\
\text { gestational age at admission }(\mathrm{PR}=2.99 ; 1.76-5.07) ; \text { cesarean section } \\
(\mathrm{PR}=2.31 ; 1.27-4.21) \text {; previous uterine scar }(\mathrm{PR}=2.57 ; 1.07-6.17) \text {. }\end{array}$ \\
\hline Cecatti et al.35 2016 & $\begin{array}{l}- \text { Brazil } \\
-27 \text { referral } \\
\text { maternities in } \\
\text { five regions }\end{array}$ & $2009-2010$ & $\begin{array}{l}\text {-"Multicenter stu- } \\
\text { dy of the National } \\
\text { Surveillance Net- } \\
\text { work on Severe } \\
\text { Maternal Morbi- } \\
\text { dity" } \\
\text {-Descriptive }\end{array}$ & $\begin{array}{l}\text {-All the } 9,555 \\
\text { women }\end{array}$ & $-\mathrm{WHO}^{3}$ & $\begin{array}{l}\text {-MNMR }=9.37 / 1,000 \text { LB; MDR }=170 / 100,000 ; \text { MNM/ MD ratio= } 5.5: 1 \text {; } \\
\text { Mortality rate }=15.4 \% \\
\text { - Hemorrhage was the most frequent for MNM and MD, in compa- } \\
\text { rison to PLTC; the same occurred for infection. Hypertension was mo- } \\
\text { re prevalent in PLTC and declined as the outcome became more seve- } \\
\text { re } \\
\text { - The most frequent Near miss criteria was management ( } 58.3 \%) \\
\text { followed by clinical }(50.3 \%) \text { and laboratorial }(50 \%) \\
\text { - In the presence of only } 1 \text { criteria, the. probability of MD was small } \\
\text { and in the presence of } 3 \text { criteria, it was high } \\
\text {-Indirect causes represented } 46 \% \text { of the maternal deaths. }\end{array}$ \\
\hline
\end{tabular}


National hospital based studies / "Born in Brazil" study and "Multicenter study of the National Surveillance Network on Severe Maternal Morbidity".

\begin{tabular}{|c|c|c|c|c|c|c|}
\hline $\begin{array}{l}\text { Author and year } \\
\text { of the article }\end{array}$ & $\begin{array}{l}\text { Study } \\
\text { location }\end{array}$ & $\begin{array}{l}\text { Studied } \\
\text { period }\end{array}$ & $\begin{array}{l}\text { Type of study/ } \\
\text { source/ analysis }\end{array}$ & Population & Criteria & Results \\
\hline $\begin{array}{l}\text { Pfitscher et al., } 43 \\
2016\end{array}$ & $\begin{array}{l}\text {-Brazil } \\
-27 \text { referral } \\
\text { maternities in } \\
\text { five regions }\end{array}$ & $2009-2010$ & $\begin{array}{l}\text { - "Multicenter stu- } \\
\text { dy of the National } \\
\text { Surveillance Net- } \\
\text { work on Severe } \\
\text { Maternal Morbi- } \\
\text { dity" } \\
\text {-Two-dimensional } \\
\text { analyses with } \\
\text { adjusted preva- } \\
\text { lence ratio calcu- } \\
\text { lated }\end{array}$ & $\begin{array}{l}\text {-Of the } 9,555 \\
\text { women, } 502 \text { had } \\
\text { severe infection }\end{array}$ & $-\mathrm{WHO}^{3}$ & $\begin{array}{l}\text { - PLTC cases: } 255 ; \text { MNM: } 182 ; M D: 65-\text { MNMR }=2.2 / 1,000 \text { LB; } \\
\text { MNMR:MD= 2.8: } 1 \text {; Mortality rate of } 26.3 \% \text {. Among the } 770 \text { MNM } \\
\text { cases. } 1 / 4 \text { was sepsis } \\
\text {-Statistically significant factors associated with SMO: Delay on care } \\
\text { (PR=1.93. } 1.36-2.74) ; \text { Spontaneous access }(P R 0.420 .29-0.59 \text { ); Previous } \\
\text { diseases (diabetes }-P R=1.82 ; \text { low weight }-P R=2.17 \text {; cancer }-P R=1.73 \\
\text { and drugs use- } P R=1.65 \text { ) }\end{array}$ \\
\hline $\begin{array}{l}\text { Pfitscher et al., } 44 \\
2016\end{array}$ & $\begin{array}{l}- \text { Brazil } \\
-27 \text { referral } \\
\text { maternities in } \\
\text { five regions }\end{array}$ & $2009-2010$ & $\begin{array}{l}\text {-"Multicenter stu- } \\
\text { dy of the National } \\
\text { Surveillance Net- } \\
\text { work on Severe } \\
\text { Maternal Morbi- } \\
\text { dity" } \\
\text {-Two-dimensional } \\
\text { analyses with } \\
\text { adjusted preva- } \\
\text { lence ratio calcu- } \\
\text { lated }\end{array}$ & $\begin{array}{l}\text { - Of the } 9,555 \\
\text { women, } 485 \text { had } \\
\text { severe respiratory } \\
\text { disorder. }\end{array}$ & $-\mathrm{WHO}^{3}$ & 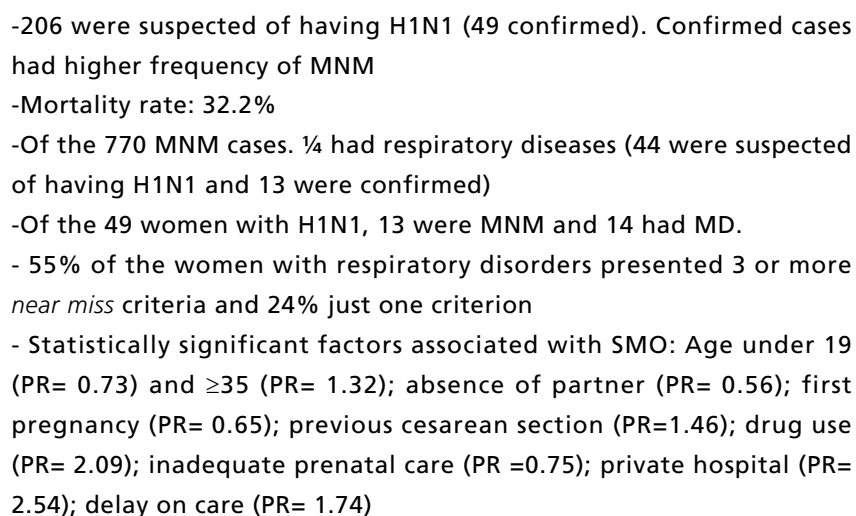 \\
\hline
\end{tabular}

PLTC (Potential life threatening conditions); SMO (Severe Maternal Outcome=MNM+MD); H1N1 (virus); HA (Hypertension); MD (maternal death); CI (confidence interval); SMM (severe maternal morbidity); MNM (Maternal Near Miss); LB (Live births); WHO (World Health Organization); OR (odds ratio); MMR (maternal mortality ratio); PR (Prevalence ratio); MNMR
(Maternal Near Miss Ratio); HDI (Human Development Index). 
The MNMR varied from 4.4/1,000 LB, according to the WHO criteria, the $188.4 / 1,000 \mathrm{LB}$, according to a criterion proposed by Reichenheim et al. ${ }^{4}$ MNM: MD ratio was 3.3 cases/ 1 death to 8.6 cases/1 death, while the mortality rate was $10.6 \%$ to $23 \%$.

The most frequent causes of MNM were the hypertensive disorders, such as severe pre-eclampsia and HELLP syndrome. The factors associated to maternal morbidity were: maternal age equal to or greater than 35 years, current or previous cesarean delivery, chronic hypertension, $<6$ pre-natal consultations.

\section{National hospital based studies}

Among hospital based articles (Table 2), 15 presented national data, all were cross-sectional studies. They were organized in two research groups. The first refers to two articles from the "Nascer no Brasil" ("Born in Brazil") study,5,34 the second reports 13 articles from the Multicenter Study of the "Rede Nacional de Vigilância de Morbidade Materna Grave"35-47 ("National Surveillance Network on Severe Maternal Morbidity”). All articles of national scope used the WHO criteria for near miss.

"Nascer no Brasil" (Born in Brazil") research was a hospital based study, covering all the Brazilian regions, but including only hospitals with more than 500 births per year and excluding cases of abortion and hospitalization during pregnancy, which did not apply to the main goal of the study. 5

The sample had 243 maternal near miss cases with an estimated 23,747 occurrences of maternal near miss in the Country, resulting in an incidence of 10.2/1,000 live births. 5 The incidence of maternal near miss was higher in women over 35 years of age, low schooling, previous cesarean history, complications during pregnancy, without prenatal care and with current cesarean section. Factors associated to statistical significance were: absence of prenatal care, obstetric complications, cesarean section and pilgrimage before the delivery. 34

The multicenter study of the Rede Brasileira de Vigilância da Morbidade Materna Grave (Brazilian Surveillance Network on Severe Maternal Morbidity) evaluated twenty-seven hospitals distributed throughout all regions of Brazil 35 between 2009 and 2010. A prospective data collection used the WHO criteria for near miss and potentially lifethreatening conditions. This research methodology was similar to most articles (Table 2).

From 82,144 deliveries with live fetuses, 9,555
$(11.6 \%)$ women were classified as having some kind of a severe outcome: $8,645(90.5 \%)$ presented severe complications, $770(8.1 \%)$ were classified as maternal near miss (WHO criteria) and 140 (1.5\%) died. ${ }^{35}$ Several articles originated from this population data, although there were no comparative studies among these 9,555 women and those who had their childbirths in the 27 units, however presenting no complications. The aspects that differ from the methodology of the study are shown in Table 2, along with each of the 13 articles. Cecatti et al. 35 present overall results, and the other articles explore MNM according to age group 36 and with specific conditions: abortion, 37 ectopic pregnancy, 38 hypertensive diseases, 39,40 hemorrhage, 41,42 infections, 43,44 heart diseases. 45 Healthcare factors were also analyzed, such as quality of care 46 and the association with cesarean section 47 based on Robson's Classification

Hypertensive disease was the main cause of maternal near miss (45\%) and maternal death (30\%), followed by hemorrhage ( $40.5 \%$ of maternal near miss and $26 \%$ of maternal deaths). More than $75 \%$ of the maternal death cases observed, more than one near miss criterion defined by WHO was found. The highest maternal near miss ratio occurred in women aged 40-49 (31.4/1,000 LB), followed by the age of 35-39 (17.5/1,000 LB), and 35-49 years old (20.55/1,000 LB), compared to adolescents with a ratio of 7.14/1,000 LB.36

\section{Population survey based studies}

Six studies (Table 3) were based on the population surveys, selecting or creating questions to enable the capture of cases during the interviews with the women. ${ }^{48-53}$

The oldest article48 used as a criterion only complications such as prolonged labor, excessive hemorrhage, high fever, seizures. The other articles ${ }^{4-53}$ used, with some adaptations, maternal conditions/complications and interventions, evaluating history of eclampsia, hysterectomy, blood transfusion and ICU admission, previously validated criteria.

Four studies relied on the 1996 and 2006 national surveys (DHS), with home interviews of women with live births in the previous five years ${ }^{48-}$ 50 and women with history of abortion. ${ }^{51}$ Cecatti et al. 52 used data from a vaccination campaign survey for women in the Amazon and the Northeast regions, while Rosendo and Roncalli53 conducted a home survey in Natal City in Rio Grande do Norte State. 
Population survey based studies.

\begin{tabular}{|c|c|c|c|c|c|c|}
\hline $\begin{array}{l}\text { Author and year } \\
\text { of the article }\end{array}$ & $\begin{array}{l}\text { Study } \\
\text { location }\end{array}$ & $\begin{array}{c}\text { Studied } \\
\text { period }\end{array}$ & $\begin{array}{l}\text { Type of study/ } \\
\text { source/ analysis }\end{array}$ & Population & Criteria & Results \\
\hline Souza et al.,48 2008 & $\begin{array}{l}\text { Brazil: seven } \\
\text { locations - São } \\
\text { Paulo, Rio de } \\
\text { Janeiro, Minas } \\
\text { Gerais and } \\
\text { Espírito Santo, } \\
\text { Midwest, South, } \\
\text { Northeast and } \\
\text { North regions }\end{array}$ & 1996 & $\begin{array}{l}\text {-Survey - DHS } 1996 \\
\text {-Household inter- } \\
\text { views in census } \\
\text { sectors } \\
\text {-Multivariate logistic } \\
\text { regression }\end{array}$ & $\begin{array}{l}-3,761 \text { women had } \\
\text { live births in the last } \\
\text { five years } \\
-5,045 \mathrm{LB}\end{array}$ & $\begin{array}{l}\text {-Complications: } \\
\text { Prolonged labor, } \\
\text { excessive hemorr- } \\
\text { hage, high fever, } \\
\text { seizures }\end{array}$ & $\begin{array}{l}\text {-Presence of complications during pregnancy: } 4.8 \% \text { prolonged labor, } \\
10.3 \% \text { hemorrhage, } 5.4 \% \text { fever, } 3.7 \% \text { seizures: } 2.7 \% \\
\text { - Significant association found with SMM: hemorrhage associated to } \\
\text { non-white skin color }(O R=1,72) \text {; any complication associated to previous } \\
\text { children }(O R=1.33) \text {, and the North }(O R=2.00) \text { and Midwest }(O R=1.33) \\
\text { regions }\end{array}$ \\
\hline Souza et al.,49 2010b & $\begin{array}{l}\text { Brazil: five } \\
\text { regions }\end{array}$ & $2006-2007$ & $\begin{array}{l}\text {-Survey - DHS } 2006 \\
\text {-Household inter- } \\
\text { views in census } \\
\text { sectors } \\
\text {-Multivariate logistic } \\
\text { regression }\end{array}$ & $\begin{array}{l}-5,025 \text { women had } \\
\text { live births in the last } \\
\text { five years } \\
-5,045 \mathrm{LB}\end{array}$ & $\begin{array}{l}\text {-Eclampsia, hyste- } \\
\text { rectomy, blood } \\
\text { transfusion and ICU } \\
\text { admission }\end{array}$ & $\begin{array}{l}\text {-Presence of complications during pregnancy: } 2 \% \text {. Most frequent } \\
\text { hemorrhage } \\
\text {-Criteria: eclampsia }-0.6 \% \text {; hysterectomy }-0.2 \% \text {; blood transfusion - } \\
0.8 \% \text { and ICU }-0.5 \% \text {. } \\
\text { - Population estimates: } \\
\text { Number of maternal near miss cases: around } 70,000 \\
\text { MNM Ratio: } 21.1 / 1,000 \text { LB } \\
\text { MNM/MD Ratio }=28.4 \text {; Mortality rate }=3.3 \% \\
\text {-Association with age } 40-49 \text { years old }(O R=9.60) \text { and low schooling } \\
(O R=2.18)\end{array}$ \\
\hline $\begin{array}{l}\text { Oliveira Jr et al.,50 } \\
2013\end{array}$ & $\begin{array}{l}\text { Brasil: cinco } \\
\text { regiões }\end{array}$ & 2006-2007 & $\begin{array}{l}\text {-Survey - DHS } 2006 \\
\text {-Household intervi- } \\
\text { ews in census sectors } \\
\text {-Multivariate logistic } \\
\text { regression }\end{array}$ & $\begin{array}{l}-5,025 \text { women had } \\
\text { live births in the last } \\
\text { five years } \\
-6,833 \text { pregnancies }\end{array}$ & $\begin{array}{l}\text {-Eclampsia, hyste- } \\
\text { rectomy, blood } \\
\text { transfusion and ICU } \\
\text { admission }\end{array}$ & $\begin{array}{l}\text {-Maternal near miss ratio by age: } \\
\text { 15-19 years: } 5.9 / 1,000 \text { LB (most frequent: eclampsia) } \\
\text { 20-34: } 19.9 / 1,000 \text { LB (most frequent: blood transfusion) } \\
\text { 35-49; 28.3/1,000 LB (most frequent: eclampsia) } \\
\text {-For age group of } 35-49 \text { years old, association with low schooling and } \\
\text { pregnancy complications (OR=5.23) }\end{array}$ \\
\hline $\begin{array}{l}\text { Camargo et al.,51 } \\
2011\end{array}$ & $\begin{array}{l}\text { Brasil: cinco } \\
\text { regiões }\end{array}$ & 2006-2007 & $\begin{array}{l}\text {-Survey - DHS } 2006 \\
\text {-Household inter- } \\
\text { views in census } \\
\text { sectors }\end{array}$ & $\begin{array}{l}\text { - 15,542 women who } \\
\text { had an abortion }\end{array}$ & $\begin{array}{l}\text {-Eclampsia; hemorr- } \\
\text { hage; infection; } \\
\text { hysterectomy; blo- } \\
\text { od transfusion; ICU } \\
\text { admission; mecha- } \\
\text { nical ventilation }\end{array}$ & $\begin{array}{l}\text {-Comparing women who had an abortion with those who had not, there } \\
\text { was a higher prevalence of hemorrhage }(P R=2.54 ; 1.85-3.49) \text {; infection } \\
(P R=2.89 ; 1.34-6.24) \text {; hysterectomy }(P R=5.37 ; 0.83-34.93) \text {, severe } \\
\text { maternal morbidity }(S M M) \text { criteria. Global association abortion } / \mathrm{SMM}= \\
2.29(1.73-3.04)\end{array}$ \\
\hline
\end{tabular}


Table 3

concluded

Population survey based studies.

Studied

Type of

study/

Author and year Study

of the article

Cecatti et al..52 -Amazônia

2015

Northeast

2010

-Secondary analysis of

the survey "Chamada childbirth history of
nom

Neonatal" performed previousyear

during vaccination

campaign

-Poisson regression

\section{Criteria}

-Eclampsia, hyste-

rectomy, blood

transfusion;

admission

ICU in RC

$\mathrm{CU}$ in $\mathrm{R}$

LB)

- Most frequent complication: hemorrhage and infection.

- Most frequent MNM criteria: eclampsia and blood transfusion

- Significant association with MNM: indigenous ethnicity ( $P R=2.77)$, prenatal care at a public health service $(P R=1.95)$, pregnancy pilgrimage $(P R=1.49)$ and cesarean childbirth $(P R=2.56)$.

Rosendo \& $\quad$-Natal, RN

2014

Roncalli,53 2015

(2014)

-Household survey
Difference in pro-
portions (Chi-square

test)
- 848 (15-49 years
nancy in the last five
years) in
8,227

blood transfusion,

ICU admission and

proposed by Souza

et al.,54)

- Maternal near miss ratio: 41.1/1,000 LB.

-Most referred criteria identified: ICU admission (19.1/1,000 LB) and eclampsia (13.5/1,000 LB), followed by blood transfusion (11.3/1,000 LB), hysterectomy $(2.3 / 1,000$ LB).

Clinical conditions mostly reported: hemorrhage $(10.7 \%)$ and urinary infection (10.7\%); most frequent intervention: hospital stay $>7$ days postpartum $(5.4 \%)$

- Increased prevalence of MNM in mixed/black women, although with significance border $(p=0.052)$

$\mathrm{DHS}=$ demographic and health survey; $\mathrm{MD}=$ maternal death; $\mathrm{SMM}=$ severe maternal morbidity; $\mathrm{MNM}=$ maternal near miss; $\mathrm{LB}=$ live births; $\mathrm{OR}=\mathrm{odd}$ ratio; $\mathrm{RN}=\mathrm{Rio} \mathrm{Grande}$ do Norte;

$\mathrm{PR}=$ prevalence ratio; ICU= intensive care unit. 
Information systems based studies on SIH-SUS and SIM.

\begin{tabular}{lccccc}
\hline $\begin{array}{l}\text { Author and, year } \\
\text { of the article }\end{array}$ & $\begin{array}{c}\text { Study } \\
\text { location }\end{array}$ & $\begin{array}{c}\text { Studied } \\
\text { period }\end{array}$ & $\begin{array}{c}\text { Type of study/ } \\
\text { source/ analysis }\end{array}$ & Population & Criteria \\
\hline
\end{tabular}

capitals and the -Descriptive diagnosis of severe -Waterstone et al.31 - The most frequent maternal near miss criteria were:

Federal District) maternal morbidity Severe

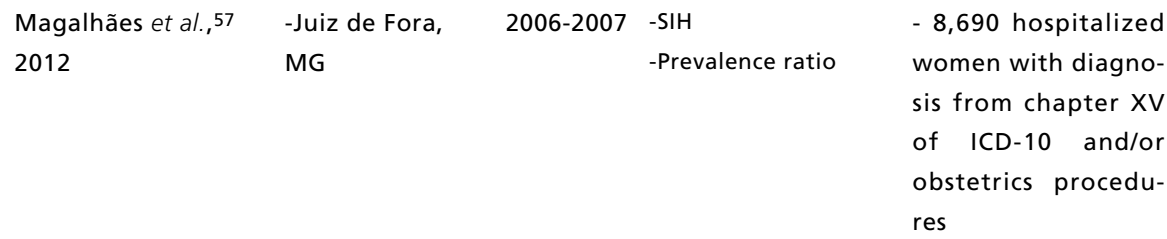

Nakamura-Pereira et -Rio de Janeiro, al., 582013 RJ
2008

- Revie

$-\mathrm{SIH} \quad-221$ cases files

-Descriptive 
Information systems based studies on SIH-SUS and SIM.

\begin{tabular}{lccccc}
\hline $\begin{array}{l}\text { Author and, year } \\
\text { of the article }\end{array}$ & $\begin{array}{c}\text { Study } \\
\text { location }\end{array}$ & $\begin{array}{c}\text { Studied } \\
\text { period }\end{array}$ & $\begin{array}{c}\text { Type of study/ } \\
\text { source/ analysis }\end{array}$ & Population & Criteria \\
\hline
\end{tabular}

Rosendo \& Roncalli, - 167 cities in 2008 -2012 -Ecologic study -Women 15-49 years Waterstone et al., 31 - MNMR average: 36.67/1,000 women (SD: 17.90). According to the

$552016 \quad$ Rio Grande do -SIH Norte, Brazil

\section{-Multip} gression

old, residents in RN,

excluding syndrome (for hemorrhage (4.55) and sepsis (4.29). Hypertensive diseases: group not having the with the highest average MNMR (27.65)

tetrics procedures at

SUS-hired or asso- corresponding ICD- - For MNMR, positive correlation with \% households with high ciated units. Cities grouped (Skater method) in 63 clusters.

10 code) density $(p=0.049)$ and $\%$ mothers head of the household with low schooling $(p=0.032)$ and negative correlation with $\%$ of families benefited by "Bolsa Familia" grant program $(p=0.001)$

- For HYPDR: positive correlation with \% households with high density $(p=0,021)$; and negative correlation with $\%$ of families benefited by "Bolsa Família" grant program $(p=0.001)$; for $\mathrm{RH}$ : positive correlation with child mortality $(p=0.041)$

- Lack of variables correlation in primary healthcare as prenatal consultations and Family Health Strategy.

Silva et al.,56 $2016 \quad$-Paraná

2010 -Descriptive

-Source: SIH

\begin{abstract}
- 111,409 women aged 10-49 years old hospitalized at SUS with pregnancy, childbirth and
puerperium complications.
\end{abstract}

Waterstone et al. 31 - Global rate of SMM: 52.9/1,000 childbirths

-Mantel et al.32 -Absolute number: 4,890 cases, 4,225 were identified by the main Established criteria diagnosis, 424 by procedures, 216 by ICU admission and 25 by by Sousa et al.54 secondary diagnosis

- Rate by age group: 43.5 for 10-19 years old, 51.6 for 20-34 years old and 88.6 for $35-49$ years old

- Rate by criteria:

Pre-eclampsia (14.9) and eclampsia (5.1); severe hemorrhage (12.5); immune disorder (7.4); severe sepsis (5.5); heart disorder (2.7) and ICU admission (2.3)

$\mathrm{PHC}=$ primary healthcare; $\mathrm{FHS}=$ family health strategy; HELLP= hemolysis elevated liver enzymes low platelet; $\mathrm{MD}=$ maternal death; $\mathrm{SMM}=$ severe maternal morbidity; $\mathrm{ESMM}=$ extremely severe maternal morbidity; $M N M=$ maternal near miss; $L B=$ live births; WHO= World Health Organization; $P N=$ prenatal; HYPDR= MNM ratio for hypertensives diseases; $H R=$ MNM ratio for hemorrhage; $M N M R=$ maternal near miss ratio; $P R=$ prevalence ratio; $R R=$ relative risk; $S I H=$ hospital information system; $S I M=m o r t a l i t y$ information system; $S U S=P u b l i c$ Health System; ICU= intensive care unit. 
The MNMR varied from 21.2/1,000 LB to 41.1/1,000 LB. Among the criteria used for MNM, the most observed were eclampsia and blood transfusion, except in the study in Natal City, where ICU hospitalization was more frequent. ${ }^{3}$ Hemorrhage was the clinical complication most commonly reported by women.

Age $\geq 35$ years old, low schooling and non-white skin color were the most frequent socioeconomic factors associated. Other cited factors were absence of prenatal and pilgrimage for childbirth.

\section{Information systems based studies}

Of the five studies (Table 4), only one evaluated the national data.54 Regarding the criteria, three $54-56$ used Waterstone and Mantel's criteria,54-56 and two 57,58 used the WHO criteria with some adaptations. Women with a history of gestation, delivery and puerperium, women diagnosed with severe maternal morbidity, and women hospitalized for obstetric procedures were included. Pre-eclampsia was the most frequent indicator by Waterstone and Mantel's criteria.

The authors used both maternal near miss, ratios varied from 32.2 and 44.4/1,000 LB, as severe/ extremely severe maternal morbidity, and the ratios varied from $36.7 / 1,000$ women in patients55 to 52.9/1,000 deliveries. 56 Nakamura-Pereira et al. 58 evaluated the information quality of the Sistema de Informações Hospitalares do Sistema Único de Saúde (SIH-SUS) (Hospital Information System of the Public Health Service) for the study on severe maternal morbidity and estimated low sensitivity (18.5\%) with high specificity $(94.3 \%)$.

In this group of studies, the worse primary care assistance, as well as the history of stillbirth children and ages between 35-49 years old were the variables associated to the poverty markers outcome.

\section{Discussion}

This review identified 48 studies on severe maternal morbidity/maternal near miss in Brazil. Unlike other themes in women and childhood health, there was a high number of studies in the Northeast region was observed.

In 2005, the first Brazilian article was published using the term maternal near miss. ${ }^{9}$ The criteria used were from Waterstone, Mantel and Geller.31-33 Hypertensive diseases and hemorrhages shared as the main causes, according to the criterion. Both conditions remain as the main causes associated to maternal morbidity.
Three approaches were adopted by the studies as regard to the data sources: ${ }^{8}$ hospital based predominance, with local or national primary or secondary data; the use of the information systems, Sistemas de Informações Hospitalares do Sistema Único de Saúde, Sistemas de Informação sobre Mortalidade (SIH-SUS, SIM) (Hospital Information System of the Public Health Service, Information System on Mortality), and also the local or national population surveys. Each one showed advantages and fragilities.

In the case of hospital based studies, the main problem concern management criteria of the WHO classification. Depending on the infrastructure, the existence of protocols and the quality of the team, the indications and applications of some procedures can vary widely among institutions. The ICU indication, alone, is already considered as a near miss criterion and is very dependent on the factors above.

Limitations for the WHO near miss criteria in case of using the SIH-SUS as a data source: difficulty in correlating these criteria with diagnoses of ICD-10 and with procedures codes adopted by SIHSUS56 and low sensitivity. 59 However, Magalhães et al. 57 used the SIH as a source and WHO criteria in their study, and evaluated the results found as satisfactory. Silva et al.56 supports the use of Waterstone's criteria for studies with SIH to increase sensitivity.

In relation to the national surveys, such as DHS, the information is self-referred and there are no forms to prove the diagnoses. Souza et al.49 comment on the limitations of the questionnaires used in this type of survey. When referring to their morbidities, women remember more of the interventions than the clinical complications; they rarely report eclampsia, for example.

The heterogeneity of the terminology was observed: Severe Maternal Morbidity, Extremely Severe Maternal Morbidity and Maternal Near Miss. In some cases, the authors use the terms indiscriminately and sometimes they use Severe Maternal Morbidity (SMM) as the synonym of Life Threatening Conditions. This heterogeneity implies the difficulty to compare results within the studies, but notice that the term maternal near miss prevailed. There was also heterogeneity in relation to the indicator that expresses the relative frequency of MNM. The MNM ratio was calculated when the denominator consisted on the number of live births and the incidence was calculated when the denominator referred to the number of childbirths or hospitalized women.

The study on maternal near miss showed the potential of indicators proposed by $\mathrm{WHO}$ as predic- 
tors of maternal death: women that presented three or more criteria were more likely to die than those that presented only one criterion. 35

There was also a similarity between the causes of MNM and those of maternal death in Brazil. Direct obstetric causes still prevail, but indirect ones are increasing. This pattern, allied to the increase of cesarean sections confirm that the Country presents itself in an obstetric transition movement. 59

The most frequently associated factors in this study with MNM/SMM/ESMM were: age $\geq 35$ years old, low schooling, current or previous cesarean section, hemorrhage, previous hypertension and prior abortion.

Inequalities inmaternal health were evidenced as regarding maternal morbidity. Considering the $\mathrm{MNM} / \mathrm{MD}$ as an indicator to assess the quality of obstetric care after women's admission, the values observed were three times lower in regions with the lowest HDI in the Country 39 In Pfitscher et al.,43 study considering that the H1N1 pandemic and its effect on maternal near miss, it was observed that non-white women progressed more frequently for more severe conditions, including death. Pacagnella et al.46 analyzed any presence of delays in pregnant women care and showed that non-white color adolescence with low schooling were strongly associated. In relation to the age group, Oliveira Jr. et al. 36 found the lowest MNMR in women aged 10 to 19 years old, however this age group had the most delays in care. The inequities in relation to these variables add to of other maternal and child health outcomes, such as prenatal access. 60

On the other hand, a protective effect was observed in relation to MNM for the beneficiary families of Bolsa Família Program55 (a Government program for extreme low income family to receive like an allowance) and the protective association of prenatal care regarding MNM was well evidenced. 18,24,28,46 It ratifies that complementing both politics and income as a basic care can reverse inequalities. However, it is necessary to improve prenatal care adequacy in SUS, considering that some studies showed worse performance compared to private prenatal care.

Brazil is known worldwide for the high incidence of cesarean sections. ${ }^{61}$ In several studies, previous or current cesarean section was associated to MNM/ESMM/SMM.21, 22, 24, 25, 27, 29, 34, 52 Only one study showed cesarean section as a protective factor. 17 The maternal near miss rate for cesarean section was 91 cases $/ 1,000$ deliveries whereas the incidence rate on vaginal delivery was $16 / 1,000$ deliveries. 32 Although it is not possible to evidence a direct association, in virtue of an adequate procedure for several gestational risk situations, even though the cesarean section rate was high and the association to postpartum hemorrhage, identified in some studies, does not justify for the prior indication but could be attributed to the procedure. Adopting Robson's classification to monitor and compare cesarean indications should be a national recommendation.

As for abortion, about $2 \%$ of the women (more than 450) interviewed in the "Born in Brazil" research reported the tentative to interrupt their current gestation. ${ }^{60}$ Souza et al. ${ }^{9}$ and Galvão et al. 22 studies found an association between previous abortion and maternal near miss, and Santana et al. 37 and Camargo et al. 51 studies reported a higher risk of near miss in women submitted to abortion.

An evaluation of national base studies is required. "Born in Brazil" research was a hospital based study covering all the Brazilian regions, including only hospitals with more than 500 childbirths per year and excluding cases of abortion and hospitalization during pregnancy which was not the main goal of this study. 5 As most births in Brazil occur in a hospital environment, this study could be considered as a population study approach, except for the above limitations. Their results, consistent with those studies at the local level have a great relevance for guiding health policies.

This study showed an association between cesarean section and maternal near miss, even after the adjustments on obstetric complications and two groups of women were identified: the first, white skin, high schooling, adequate prenatal care, no history of pilgrimage for delivery and a high frequency of elective cesarean section; and the second one, mixed or black skin, low schooling, younger, with absence of prenatal care and a higher frequency of vaginal delivery.

Domingues et al. 34 concluded that indiscriminate use of cesarean section may approximate the occurrence of MNM between the two groups. However, if the interpretation of the hierarchical model was used, preserving distal factors without adjustment, there was a positive association between age $\geq 35$ years old, low schooling, primiparity and previous cesarean section (considering the OR obtained in model 1, without adjusting for intermediate and distal variables). This analysis reinforces inequality in near miss occurrence and the importance of previous cesarean section.

Studies derived from the Multicenter Surveillance Network for Severe Maternal Morbidity explored for the first time MNM 
subgroups according to different conditions and increasing the knowledge on maternal morbidity. Maternity hospitals were select from five Brazilian regions, with a greater representation in the Southeast, mainly São Paulo State. As a limitation, the maternities included were predominantly tertiary/reference and were not representative of the morbidity population profile. This is confirmed by the MMR found in the study of 170/100,000 live births. However, they managed to capture in these locations a large number of patients with severe maternal morbidity, increasing statistical power of the results.

Another limitation was the absence of comparison with the control group (women without complications in the maternity hospitals), which does not allow to identify the population risk factors. In contrast, it was possible to evaluate the severity gradient in the subgroups in the same clinical condition, observing the relationship between PLTC, MNM and MD. The highest mortality rate was found for respiratory diseases (32.2\%), with emphasis on H1N1 (51.8\%), followed by infections (26.3\%), heart diseases $(24 \%)$, eclampsia (19\%), postpartum hemorrhage (15\%), hypertensive disease $(10.7 \%)$, and antepartum hemorrhage (9\%). Although the mortality rate for hemorrhagic conditions is not so high, the frequency of placental abruption is high, contributing for near miss. When the data was collected for this study, there was a H1N1 pandemic underway (2009) and the severity of this infection in pregnant women revealed high morbidity and mortality.

When comparing these two studies, a very similar MNM ratio was observed, but the MMR was much higher in the Multicenter Network study. In addition to the reasons explained above, "Born in Brazil" study did not directly estimate maternal deaths, but used a proxy, which may have contributed to the difference found. Regardless to the differences between the studies, several results are similar and corroborate the need to increase and qualify care for women.

There is a predominance of publications in international journals in the English language, which may hampered the dissemination of the near miss concept among health professionals in our Country. In international journals, the area of Reproductive Health was predominant, while in national journals, public health showed to be more productive and there were only three articles in the journals of the GynecologyObstetrics specialty.

As the limitations of this review, we point out the bibliographic search, which unpublished studies were not included. We only used well-known keywords used by WHO: near miss and severe maternal morbidity. However, we believe that we have made our search more specific, considering that many national studies have already incorporated the WHO terminology. We would like to suggest the incorporation of the terms to the health descriptors.

Regarding possible information bias, the reading and extraction of the data by more than one researcher, independently, contributed to its attenuation.

There are no other systematic national reviews on the subject, and the most recent review included a few Brazilian studies. 6 And besides that, this study goes back to prior studies conducted before the WHO definition for near miss. Therefore, comparison is limited. Nevertheless, we highlight the heterogeneity of the near miss criteria, the prevalence of hospital based studies and the presence of social inequalities.

The study on maternal morbidity has been relevant in Brazil in pointing out fragile points in the health services. Although the results vary, the frequency of women with potential life-threatening complications is high in Brazil, which reinforces the need to universalize more complex interventions as well as coverage of primary care. 62

We conclude that the evaluation of maternal near miss should be implanted as a routine in the maternity hospitals, using the WHO criteria of greater specificity and adding other criteria according to the capacity of each unit, in increasing sensitivity. It is important to emphasize that it should not only be an aid for the study on maternal mortality, but also for the conditions of maternal morbidity in pregnancy, childbirth and the puerperium.

In the research field, the theme is not exhausted; other studies evaluating more than one criterion and using longitudinal outlines are necessary to deepen the understanding of maternal morbidity and mortality in Brazil. 


\section{References}

1. United Nations. Sustainable Development Goals [Internet] New York: United Nations; 2017. [acesso em 26 set 2017] Disponível em: https://sustainabledevelopment.un.org/sdg3

2. Say L, Barreix M, Chou D, Tunçalp Ö, Cottler S, McCawBinns A, Gichuhi GN, Taulo F, Hindin M. Maternal morbidity measurement tool pilot: study protocol. Reprod Health. 2016; 13 (1): 69.

3. Organização Mundial de Saúde. Avaliação da Qualidade do Cuidado nas Complicações Graves da Gestação: A Abordagem do Near Miss da OMS para a Saúde Materna. Uruguay. OMS; 2011.

4. Reichenheim ME, Zylbersztajn F, Moraes CL, Lobato G. Severe acute obstetric morbidity (near miss): a review of the relative use of its diagnostic indicators. Arch Gynecol Obstet. 2009; 280 (3): 337-43.

5. Dias MAB, Domingues RMSM, Schilithz AOC, Pereira MN, Diniz CSG, Brum IR, Martins AL, Theme Filha MM, Gama SGN, Leal MC. Incidência do Near Miss Materno no Parto e Pós-parto hospitalar: dados da pesquisa Nascer no Brasil. Cad Saúde Pública. 2014; 30 (Supl 1): S1-12.

6. Tunçalp Ö, Hindin MJ, Souza JP, Chou D, Say L. The prevalence of maternal near miss: a systematic review. BJOG. 2012; 119 (6): 653-61

7. Liberati A, Altman DG, Tetzlaff J, Mulrow C, Gotzsche PC, Loannidis JPA, Clarke M, Devereau PJ, Kleijnen J, Moher D. The PRISMA statement for reporting systematic reviews and meta-analyses of studies that evaluate healthcare interventions: explanation and elaboration. BMJ 2009; 339 b2700.

8. Cecatti JG, Souza JP, Parpinelli MA, de Sousa MH, Amara E. Research on Severe Maternal Morbidities and NearMisses in Brazil: What We Have Learned. Reprod Health Matters. 2007; 15 (30): 125-33

9. Souza JPD, Cecatti JG, Parpinelli MA. Fatores associados à gravidade da morbidade materna na caracterização do near miss. Rev Bras Ginecol Obstet. 2005; 27 (4): 197-203.

10. Souza JP, Cecatti JG, Parpinelli MA, Serruya SJ, Amaral E. Appropriate criteria for identification of near miss maternal morbidity in tertiary care facilities: a cross sectional study. BMC Pregnancy Childbirth. 2007; 11 (7): 20.

11. Amorim MM, Katz L, Valença M, Araújo DE. Morbidade materna grave em UTI obstétrica no Recife, região nordeste do Brasil. Rev Assoc Med Bras. 2008; 54 (3): 261-6.

12. Luz AG, Tiago DB, Silva JC, Amaral E. Severe maternal morbidity at a local reference university hospital in Campinas, São Paulo, Brazil. Rev Bras Ginecol Obstet. 2008; 30 (6): 281-6.

13. Oliveira Neto AF, Parpinelli MA, Cecatti JG, Souza JP, Sousa MH. Factors associated with maternal death in women admitted to an intensive care unit with severe maternal morbidity. Int J Gynaecol Obstet. 2009; 105 (3) 252-6.

14. Amaral E, Souza JP, Surita F, Luz AG, Sousa MH, Cecatti JG, Campbell O. A population-based surveillance study on severe acute maternal morbidity (near miss) and adverse perinatal outcomes in Campinas, Brazil: the Vigimoma Project. BMC Pregnancy Childbirth. 2011; 11:9
15. Morse ML, Fonseca SC, Gottgtroy CL, Waldmann CS, Gueller E. Morbidade Materna Grave e Near Miss em Hospital de Referência Regional. Rev Bras Epidemiol. 2011; 14 (2): 310-22.

16. Moraes AP, Barreto SM, Passos VM, Golino PS, Costa JA, Vasconcelos MX. Incidence and main causes of severe maternal morbidity in São Luís, Maranhão, Brazil: a longitudinal study. São Paulo Med J. 2011; 129 (3): 146-52.

17. Lotufo FA, Parpinelli MA, Haddad SM, Surita FG, Cecatti JG. Applying the new concept of maternal near miss in an intensive care unit. Clinics. 2012; 67 (3): 225-30.

18. Moraes APP, Barreto SM, Passos VM a, Golino PS, Costa JE, Vasconcelos MX. Severe maternal morbidity: a casecontrol study in Maranhão, Brazil. Reprod Health. 2013; 10:11.

19. Lobato G, Nakamura-Pereira M, Mendes-Silva W, Dias MAB, Reichenheim ME. Comparing different diagnostic approaches to severe maternal morbidity and near miss: a pilot study in a Brazilian tertiary hospital. Eur J Obstet Gynecol Reprod Biol. 2013; 167 (1): 24-8.

20. Oliveira LC, Costa AAR. Óbitos Fetais e Neonatais entre Casos de Near Miss Materno. Rev Assoc Med Bras. 2013; 59 (5): 487-94.

21. Amorim MM, Katz L, Barros AS, Almeida TS, Souza AS, Faúndes A. Maternal outcomes according to mode of delivery in women with severe preeclampsia: a cohort study. J Matern Fetal Neonatal Med. 2014; 28 (6): 654-60.

22. Galvão LP, Alvim-Pereira F, de Mendonça CM, Menezes FE, Góis KA, Ribeiro RF, Gurgel RQ. The prevalence of severe maternal morbidity and near miss and associated factors in Sergipe, Northeast Brazil. BMC Pregnancy Childbirth. 2014; 14: 25. doi:10.1186/1471-2393-14-25

23. Menezes FE, Galvão LP, de Mendonça CM, Góis KA, Ribeiro RF Jr, Santos VS, Gurgel RQ. Similarities and differences between WHO criteria and two other approaches for maternal near miss diagnosis. Trop Med Int Health. 2015; 20 (11): 1501-06.

24. Pacheco AJ, Katz L, Souza AS, de Amorim MM. Factors associated with severe maternal morbidity and near miss in the São Francisco Valley, Brazil: a retrospective, cohort study. BMC Pregnancy Childbirth. 2014; 14: 91.

25. Souza MAC, Souza TH, Gonçalves AK. Fatores determinantes do near miss materno em uma unidade de terapia intensiva obstétrica. Rev Bras Ginecol Obstet. 2015; 37 (11): 498-504.

26. Oliveira LC, Costa AAR. Near miss materno em unidade de terapia intensiva: aspectos clínicos e epidemiológicos. Rev Bras Ter Intensiva. 2015; 27 (3): 220-7

27. Madeiro AP, Cronemberger AR, Lacerda EZG, Brasil LG. Incidence and determinants of severe maternal morbidity: a transversal study in a referral hospital in Teresina, Piauí, Brazil. BMC Pregnancy and Childbirth. 2015, 15: 210.

28. Barbosa IRC, Silva WBM, Cerqueira GSG, Novo NF, Almeida FA, Novo JLVG. Maternal and fetal outcome in women with hypertensive disorders of pregnancy: the impact of prenatal care. Ther Adv Cardiovasc Dis. 2015; 9 (4): $140-6$. 
29. Ferreira EC, Pacagnella RC, Costa ML, Cecatti JG. The Robson ten-group classification system for appraising deliveries at a tertiary referral hospital in Brazil. Int $\mathbf{J}$ Gynaecol Obstet. 2015a; 129 (3): 236-9.

30. Vidal, CE, Carvalho MAB, Grimaldi IR, Reis MC; Baêta MCN, Garcia RB, Silva SAR. Morbidade materna grave na microrregião de Barbacena/MG. Cad Saúde Coletiva. 2016; 24 (2): 131-8.

31. Waterstone M, Bewley S, Wolfe C. Incidence and predictors of severe obstetric morbidity: case-control study. BMJ. 2001;322(7294):1089-93

32. Mantel GD, Buchmann E, Rees H, Pattinson RC. Severe acute maternal morbidity: a pilot study of a definition for a near miss. Br J Obstet Gynaecol. 1998; 105 (9): 985- 90.

33. Geller SE, Rosenberg D, Cox SM, Kilpatrick S. Defining a conceptual framework for near miss maternal morbidity. J Am Med Womens Assoc. 2002; 57 (3): 135-9.

34. Domingues RM, Dias MA, Schilithz AO, Leal MD. Factors associated with maternal near miss in childbirth and the postpartum period: findings from the birth in Brazil National Survey, 2011-2012. Reprod Health. 2016; 13(Suppl 3): 115.

35. Cecatti JG, Costa ML, Haddad SM, Parpinelli MA, Souza JP, Sousa MH, Surita FG, Pinto e Silva JL, Pacagnella RC, Passini Jr R. Network for Surveillance of Severe Maternal Morbidity study Group. Network for Surveillance of Severe Maternal Morbidity: a powerful national collaboration generating data on maternal health outcomes and care BJOG. 2016; 123 (6): 946-53

36. Oliveira Jr FC, Surita FG, Pinto e Silva JL, Cecatti JG, Parpinelli MA, Haddad SM, Costa ML, Pacagnella RC, Sousa MH, Souza JP. Severe maternal morbidity and maternal near miss in the extremes of reproductive age: results from a national cross-sectional multicenter study. BMC Pregnancy Childbirth. 2014; 14: 77.

37. Santana DS, Cecatti JG, Parpinelli MA, Haddad SM, Costa ML, Sousa MH, Souza JP, Camargo RS, Pacagnella RC, Surita FG, Pinto e Silva JL. Brazilian Network for Surveillance of Severe Maternal Morbidity. Severe maternal morbidity due to abortion prospectively identified in a surveillance network in Brazil. Int J Gynaecol Obstet. 2012; 119 (1): 44-8.

38. Rocha Filho EA, Santana DS, Cecatti JG, Costa ML, Haddad SM, Parpinelli MA, Sousa MH, Camargo RS, Pacagnella RC, Surita FG, Pinto e Silva JL. Awareness about a life-threatening condition: ectopic pregnancy in a network for surveillance of severe maternal morbidity in Brazil. BioMed Res Int. 2014; 965724.

39. Giordano JC, Parpinelli MA, Cecatti JG, Haddad SM, Costa ML, Surita FG, Pinto e Silva JL, Sousa MH. The burden of eclampsia: results from a multicenter study on surveillance of severe maternal morbidity in Brazil. PLoS One. 2014; 9 (5): e97401.

40. Zanette E, Parpinelli MA, Surita FG, Costa ML, Haddad SM, Sousa MH, Pinto e Silva JL, Souza JP, Cecatti JG. Brazilian Network for Surveillance of Severe Maternal Morbidity Group. Maternal near miss and death among women with severe hypertensive disorders: a Brazilian multicenter surveillance study. Reprod Health. 2014; 11 (1):
41. Rocha Filho EA, Costa ML, Cecatti JG, Parpinelli MA, Haddad SM, Sousa MH, Melo JR EF, Surita FG, Souza JP. Contribution of antepartum and intrapartum hemorrhage to the burden of maternal near miss and death in a national surveillance study. Acta Obstet Gynecol Scand. 2015; 94(1): 50-8

42. Rocha Filho EA, Costa ML, Cecatti JG, Parpinelli MA, Haddad SM, Pacagnella RC, Sousa MH, Melo Jr EF, Surita FG, Souza JP. Brazilian Network for Surveillance of Severe Maternal Morbidity Study Group. Severe maternal morbidity and near miss due to postpartum hemorrhage in a national multicenter surveillance study. Int J Gynaecol Obstet. 2015; 128 (2): 131-6

43. Pfitscher LC, Cecatti JG, Pacagnella RC, Haddad SM, Parpinelli MA, Souza JP, Quintana SM, Surita FG, Costa ML. Brazilian Network for Surveillance of Severe Maternal Morbidity Group. Severe maternal morbidity due to respiratory disease and impact of $2009 \mathrm{H} 1 \mathrm{~N} 1$ influenza A pandemic in Brazil: results from a national multicenter cross-sectional study. BMC Infect Dis. 2016a; 16: 220.

44. Pfitscher LC, Cecatti JG, Haddad SM, Parpinelli MA, Souza JP, Quintana SM, Surita FG, Costa ML The role of infection and sepsis in the Brazilian Network for Surveillance of Severe Maternal Morbidity. Trop Med Int Health. 2016; 21 (2): 183-93.

45. Campanharo FF, Cecatti JG, Haddad SM, Parpinelli MA, Born D, Costa ML, Mattar R; The Impact of Cardiac Diseases during Pregnancy on Severe Maternal Morbidity and Mortality in Brazil. PLoS One. 2015; 10 (12): e0144385.

46. Pacagnella RC, Cecatti JG, Parpinelli MA, Sousa MH, Haddad SM, Costa ML, Souza JP, Pattinson RC. Delays in receiving obstetric care and poor maternal outcomes: results from a national multicentre cross-sectional study. BMC Pregnancy Childbirth. 2014; 5 (14): 159.

47. Ferreira EC, Costa ML, Cecatti JG, Haddad SM, Parpinelli MA, Robson MS. Robson Ten Group Classification System applied to women with severe maternal morbidity. Birth. 2015; 42 (1): 38-47.

48. Souza JP, Souza MH, Parpinelli MA, Amaral E, Cecatti JG. Self-reported maternal morbidity and associated factors among Brazilian women. Rev Assoc Med Bras. 2008; 54 (3): 249-55.

49. Souza JP, Cecatti JG, Parpinelli M, Sousa MH, Lago TG, Pacagnella RC, Camargo RS. Maternal morbidity and near miss in the community: findings from the 2006 Brazilian demographic health survey. BJOG. 2010; 117 (13): 158692.

50. Oliveira Jr FC, Costa ML, Cecatti JG, Pinto e Silva JL, Surita FG. Maternal morbidity and near miss associated with maternal age: the innovative approach of the 2006 Brazilian demographic health survey. Clinics. 2013; 68 (7): 922-7.

51. Camargo RS, Santana DS, Cecatti JG, Pacagnella RC, Tedesco RP, Melo EF Jr, Sousa MH. Severe maternal morbidity and factors associated with the occurrence of abortion in Brazil. Int J Gynaecol Obstet. 2011; 112 (2): 8892. 
52. Cecatti JG, Souza RT, Pacagnella RC, Leal MC, Moura EC, Santos LM. Maternal near miss among women using the public health system in the Amazon and Northeast regions of Brazil. Rev Panam Salud Pública. 2015; 37 (4-5): 232-8.

53. Rosendo TM, Roncalli AG. Prevalência e fatores associados ao Near Miss Materno: inquérito populacional em uma capital do Nordeste Brasileiro. Ciênc Saúde Colet. 2015; 20 (4): $1295-304$

54.Sousa MH, Cecatti JG, Hardy EE, Serruya SJ. Severe maternal morbidity (near miss) as a sentinel event of maternal death. An attempt to use routine data for surveillance. Reprod Health. 2008; $5: 6$.

55. Rosendo TM, Roncalli AG. Near miss materno e iniquidades em saúde: análise de determinantes contextuais no Rio Grande do Norte, Brasil. Ciênc Saúde Colet. 2016; 21 (1): 191-201.

56. Silva TC, Varela PLR, Oliveira RR, Mathias TAF Morbidade materna grave identificada no Sistema de Informações Hospitalares do Sistema Único de Saúde, no estado do Paraná, 2010. Epidemiol Serv Saúde. 2016; 25 (3): 617-28.

57. Magalhães MC, Bustamante-Teixeira MT. Morbi $\neg$ dade materna extremamente grave: uso do Sistema de Informação Hospitalar. Rev Saúde Pública. 2012; 46 (3): 472-78

58. Nakamura-Pereira M, Mendes-Silva W, Dias MAB, Reichenheim ME, Gustavo Lobato. Sistema de In $\neg$ formações Hospitalares do Sistema Único de Saúde (SIH-SUS): uma avaliação do seu desempenho para a identificação do near miss materno. Cad Saúde Pública. 2013; 29 (7): 1333-45.

Received on May 05, 2017

Final version presented on October 27, 2017

Approved on February 09, 2018
59. Souza JP, Tunçalp Ö, Vogel JP, Bohren M, Widmer M, Oladapo OT, Say L, Gülmezoglu AM, Temmerman M. Obstetric transition: the pathway towards ending preventable maternal death. BJOG. 2014; 121 (Suppl 1): 1-

60. Viellas EF, Domingues RMSM, Domingues RMSM, Dias MAB, da Gama SGN, Theme Filha MM, Costa JV, Bastos MH, Leal MC. Assistência pré-natal no Brasil. Cad Saúde Pública. 2014; 30: S85-100.

61. Barros FC, Matijasevich A, Maranhão AGK, Escalante Juan JJ, Rabello Neto DL, Fernandes RM, Vilella MEA, Matos AC; Albuquerque C; Léon RGP, Victora CG. Cesarean sections in Brazil: will they ever stop increasing? Rev Panam Salud Pública. 2015; 38 (3): 217-25.

62. Souza JP, Gülmezoglu AM, Vogel J, Carroli G, Lumbiganon P, Qureshi Z, Costa MJ, Fawole B, Mugerwa Y, Nafiou I, Neves I, Wolomby-Molondo JJ, Bang HT, Cheang K, Chuyun K, Jayaratne K, Jayathilaka CA, Mazhar SB, Mori R, Mustafa ML, Pathak LR, Perera D, Rathavy T, Recidoro Z, Roy M, Ruyan P, Shrestha N, Taneepanichsku S, Tien NV, Ganchimeg T, Wehbe M, Yadamsuren B, Yan W, Yunis K, Bataglia V, Cecatti JG, Hernandez-Prado B, Nardin JM, Narváez A, Ortiz-Panozo E, Pérez-Cuevas R, Valladares E, Zavaleta N, Armson A, Crowther C, Hogue C, Lindmark G, Mittal S, Pattinson R, Stanton ME, Campodonico L, Cuesta C, Giordano D, Intarut N, Laopaiboon M, Bahl R, Martines J, Mathai M, Merialdi M, Say L. Moving beyond essential interventions for reduction of maternal mortality (the WHO Multicountry Survey on Maternal and Newborn Health): a cross-sectional study. Lancet. 2013; 381 (9879): 1747-55. 